\title{
Analytical bunch compression studies for a linac-based electron accelerator
}

\author{
M. Schreck" \\ Laboratory for Applications of Synchrotron Radiation, Karlsruhe Institute of Technology-Campus South, \\ 76131 Karlsruhe, Germany and Indiana University Center for Spacetime Symmetries, \\ Indiana University, Bloomington, Indiana 47405-7105, USA
}

P. Wesolowski ${ }^{\dagger}$

ANKA Synchrotron Radiation Facility, Karlsruhe Institute of Technology-Campus North, 76344 Eggenstein-Leopoldshafen, Germany

(Received 7 July 2014; revised manuscript received 8 May 2015; published 19 October 2015)

\begin{abstract}
The current paper deals with analytical bunch compression studies for FLUTE whose results are compared to simulations. FLUTE is a linac-based electron accelerator with a design energy of approximately $40 \mathrm{MeV}$ currently being constructed at the Karlsruhe Institute of Technology. One of the goals of FLUTE is to generate electron bunches with their length lying in the femtosecond regime. In the first phase this will be accomplished using a magnetic bunch compressor. This compressor forms the subject of the studies presented. The paper is divided into two parts. The first part deals with pure geometric investigations of the bunch compressor where space charge effects and the backreaction of bunches with coherent synchrotron radiation are neglected. The second part is dedicated to the treatment of space charge effects. The upshot is that the analytical results in the two parts agree quite well with what is obtained from simulations. This paper shall form the basis for future analytical studies of the FLUTE bunch compressor and of bunch compression, in general.
\end{abstract}

DOI: 10.1103/PhysRevSTAB.18.100101

PACS numbers: 29.20.Ej, 29.27.-a, 41.75.Ht, 41.85.-p

\section{INTRODUCTION}

FLUTE is a linac-based electron accelerator which is presently being built at the ANKA Synchrotron Radiation Facility at the Karlsruhe Institute of Technology [1-12]. The acronym FLUTE stands for the German expression Ferninfrarot Linac- Und Test-Experiment translated to English as "Far-infrared Linac- and Test Experiment." FLUTE has a design energy of approximately $40 \mathrm{MeV}$ where the baseline machine layout of the first phase is depicted in Fig. 1.

In the current design the electron source is a $21 / 2$ cell photocathode radiofrequency (rf) gun with a maximum repetition rate of $10 \mathrm{~Hz}$. Electrons are emitted by shooting a pulsed Ti:Sa laser with a fundamental wavelength of $800 \mathrm{~nm}$ on a copper cathode where its third frequency harmonic will be used. The released electrons are then accelerated to $7 \mathrm{MeV}$. The charge of the bunches produced by the gun is planned to range from $1 \mathrm{pC}$ to $3 \mathrm{nC}$. Upon leaving the gun the beam is transversally focused by a solenoid before entering the linac accelerating the electrons

\footnotetext{
Corresponding author. mschreck@indiana.edu

pawel.wesolowski@kit.edu

Published by the American Physical Society under the terms of the Creative Commons Attribution 3.0 License. Further distribution of this work must maintain attribution to the author $(s)$ and the published article's title, journal citation, and DOI.
}

to the design energy of approximately $40 \mathrm{MeV}$. Behind the linac the beam is focused again by a doublet of quadrupole magnets before it enters the bunch compressor consisting of four dipole magnets.

One goal of FLUTE is to produce coherent synchrotron radiation (CSR) in the terahertz $(\mathrm{THz})$ range. To achieve this, subpicosecond bunch lengths will be necessary where the aim is to compress bunches to lengths in the femtosecond regime. For the past few years there has been a growing interest in coherent $\mathrm{THz}$ sources due to the various possibilities of using this kind of radiation both in research and in application. The following four paragraphs do not claim to be complete but will give some representative examples.

In Ref. [13] it was shown theoretically that by applying an external oscillating electric field to a sample of graphene, it is possible to produce higher harmonic modes. At room temperature this effect may occur for frequencies in the $\mathrm{THz}$ regime. Therefore it could open the way to graphene devices in $\mathrm{THz}$ electronics.

In a cuprate superconductor a special kind of soliton was excited successfully by using intense and narrow-band $\mathrm{THz}$ radiation [14]. If the generation, acceleration, and stopping of such solitons is under control, these could be exploited for transporting and storing information in such composites.

The chemical composition $\mathrm{BaTiO}_{3}$ is ferroelectric, i.e., below some critical temperature it exhibits domains with a spontaneous electric dipole moment. These domains are 


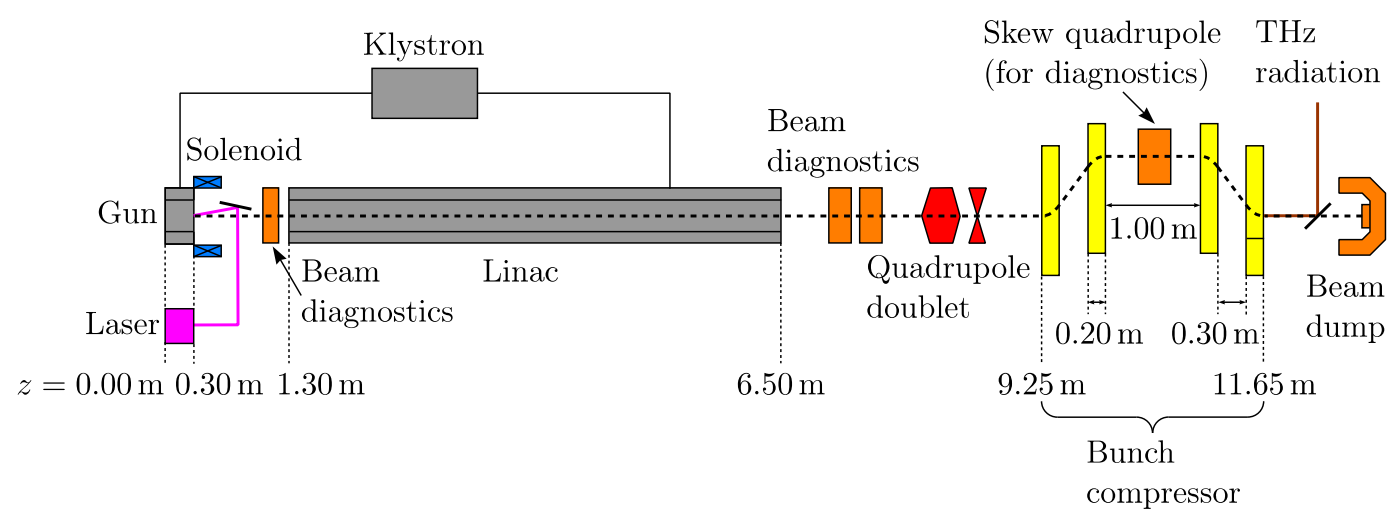

FIG. 1. Baseline layout of FLUTE in the first phase, where the position of the various parts of the machine are shown on the $z$-axis. The dashed line is the trajectory of an electron bunch. Such bunches are produced in a photocathode gun and accelerated by the linac to the design energy of $40 \mathrm{MeV}$. The rf of $3 \mathrm{GHz}$ for the gun and the linac is delivered by a klystron. Solenoids and quadrupole magnets are used to focus the beam in the transverse directions. We plan to place diagnostics at certain positions along the machine to extract information on the transverse and longitudinal beam dimensions. Electron bunches are supposed to be compressed by a bunch compressor consisting of four rectangular dipole magnets. After compressing, the bunches produce coherent THz radiation that is coupled out before the electrons hit the beam dump.

separated by domain walls that can be manipulated by applying a strong, external electric field. In Ref. [15] the physical mechanisms occurring at microscopic scales are investigated and the results are compared with experimental data. If the microscopic mechanisms of moving domain walls are better understood, such ferroelectric materials could be the basis for ultrafast computer memories.

By experiment it was shown that the magnetization direction of thin cobalt films can be reversed by short $\mathrm{THz}$ pulses, if the magnetization vector lies in the plane of the film [16]. Some (but not all) characteristics of the experimental results can be described by a simple model based on the Landau-Lifshitz equation. A better understanding of the physics and a further development of this method could lead to novel devices used for magnetic recording at high data rates.

The applications have two characteristics in common: they need high electric and magnetic field strengths (in the order of magnitude of $\mathrm{MV} / \mathrm{m}$ and several hundred $\mathrm{kA} / \mathrm{m}$, respectively) and they happen on ultrashort time scales (picoseconds). These properties can be provided by pulses of coherent synchrotron radiation in the THz regime (see, e.g., [4]).

In FLUTE the compression of the electron bunches shall be achieved with a magnetic bunch compressor. This compressor is a D-shape chicane consisting of four dipole magnets with each of them having the same magnetic field strength value. The directions of the field in the first and fourth dipole magnet are opposite to the directions in the second and third magnet. The distances between the first two and the last two magnets are supposed to be equal.

Since the electrons travel on curved trajectories inside this chicane they emit synchrotron radiation. If the bunch length is much smaller than the wavelength of the radiation, wave trains emitted from different electrons are in phase with respect to each other and they can interfere constructively. The radiation produced is then coherent and its intensity grows with the number of radiating electrons squared. Hence, the FLUTE chicane serves the purpose of compressing the bunches and is the place where the coherent radiation will be generated.

Due to space charge effects and the self-interaction of bunches with their own coherent radiation field a compression of bunches to a length of several femtoseconds is a challenging task - not only for FLUTE but for a number of other future machines as well. That is why a better understanding of the chicane is of paramount importance. Therefore, the scope of the current paper is to provide a framework for analytical bunch compression studies for FLUTE. The analytical results will also be compared to results obtained with the simulation tool Astra [17].

The paper is organized as follows. In Sec. II bunch compression by path length differences in the FLUTE chicane is discussed. Those studies are restricted to the pure geometrical properties of the chicane where space charge forces and the emission of CSR is neglected. Subsequently the transfer matrix method is employed in Sec. III to describe bunch compression and the results are compared to the outcome of simulations. In this context a number of hitherto unknown transfer coefficients at first- and second-order perturbation theory is derived. Section IV is dedicated to investigating bunch compression with space charge effects switched on. We introduce a simple model to describe space charge forces and compare our results with simulations again. Finally we conclude on the results in Sec. V. Calculational details are relegated to Appendixes A and B.

\section{BUNCH COMPRESSION BY PATH LENGTH DIFFERENCES}

In the current section analytical results on bunch compression in the FLUTE chicane are obtained, where a draft 


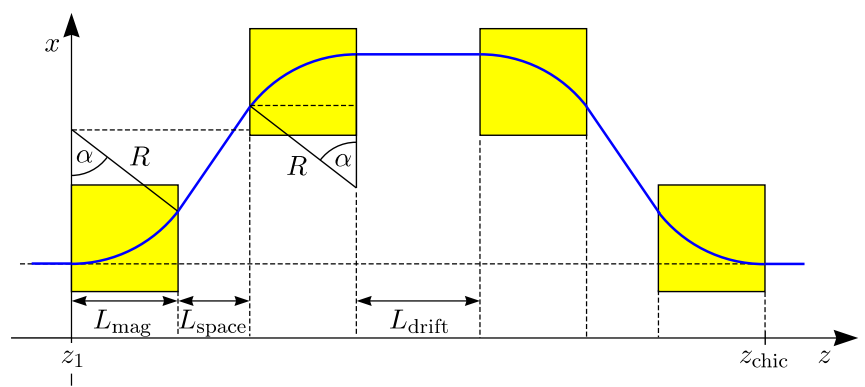

FIG. 2. Draft of the D-shape chicane foreseen for FLUTE. A Cartesian coordinate system is used where its labels $x$ and $y$ (orthogonal to the drawing plane) correspond to the two transverse directions and the label $z$ corresponds to the longitudinal direction. The chicane is assumed to lie in the $x-z$-place and the $z$-axis points along the direction of the electron beam right before the chicane. The plain (blue) curve depicts one possible electron trajectory. The length of a single chicane magnet is denoted as $L_{\text {mag. }}$. The distance between the first two and the last two magnets is called $L_{\text {space}}$, whereas the distance between the second and the third magnet is denoted as $L_{\text {drift }}$. The angle $\alpha$ is the bending angle of each magnet and $R$ is the bending radius.

of the latter is shown in Fig. 2. To make this approach feasible, the D-shape chicane is considered to consist of ideal dipole magnets. These are assumed to have a homogeneous magnetic field with flux density $B$ inside the poles which immediately drops to zero outside. In the first and fourth magnet the field is to point along the negative $y$-axis, whereas in the second and third magnet it points along the positive $y$-axis.

The bending radius in a chicane magnet is given by $R=p /(e \boldsymbol{B})$, where $p=\gamma(v) m_{\mathrm{e}} v$ is the relativistic electron momentum with the Lorentz factor

$$
\gamma(v)=\frac{1}{\sqrt{1-\beta^{2}}}, \quad \beta=\frac{v}{c} .
$$

Here $m_{\mathrm{e}}$ is the electron rest mass, $v$ the electron propagation velocity, and $c$ the speed of light. An electron has the charge $q=-e$ with the elementary charge $e>0$. The bending angle can be computed as $\alpha=\arcsin \left(L_{\mathrm{mag}} / R\right)$.

First of all, space charge effects and the backreaction of the bunch with its CSR will be neglected. As a result, all considerations of the current chapter are of geometrical nature. The reduction of the bunch length within the chicane then essentially results from the path length difference of electrons with different momenta. The length of the trajectory of an electron traveling with momentum $p$ is given by

$$
\begin{aligned}
L(p) & =4 R \arcsin \left(\frac{L_{\mathrm{mag}}}{R}\right)+\frac{2 L_{\mathrm{space}}}{\sqrt{1-\left(L_{\mathrm{mag}} / R\right)^{2}}}+L_{\mathrm{drift}}, \\
R & =\frac{p}{e B} .
\end{aligned}
$$

Now the difference between the traveling lengths of two electrons is considered. The first electron is assumed to travel with the design (reference) momentum $p$ and the second electron with a momentum that deviates from $p$ by $\Delta p$. For $\Delta p \ll p$ a Taylor expansion can be performed with respect to the dimensionless normalized momentum deviation $\delta \equiv \Delta p / p \ll 1$. Due to the limited extension of the beam pipe, the bending angle $\alpha$ must be much smaller than $\pi / 2$. This translates to the necessary condition that $L_{\text {mag }} \ll R$. Hence, it makes sense to perform a second expansion with respect to the small ratio $L_{\mathrm{mag}} / R$. That leads to a transparent result for the path length difference:

$$
\begin{aligned}
\Delta L & \equiv L(p+\Delta p)-L(p) \\
& =-2\left(\frac{L_{\mathrm{mag}}}{R}\right)^{2}\left[\frac{2}{3} L_{\mathrm{mag}}+L_{\text {space }}\right] \delta+\mathcal{O}\left[\delta^{2},\left(L_{\mathrm{mag}} / R\right)^{4}\right] .
\end{aligned}
$$

It is evident that $\Delta L<0$ for $\delta>0$. This is clear since the bending angle of an electron with a larger momentum is smaller resulting in a shorter path length traveled by the corresponding particle.

We decided to perform the following calculations throughout the paper for the two extreme beam dynamics cases that were simulated with Astra: a bunch with the high charge of $3 \mathrm{nC}$ and a bunch with the very low charge of $1 \mathrm{pC}$.

\section{A. Electron trajectory inside the chicane}

The longitudinal phase space distribution of electron bunches produced at FLUTE, i.e., their longitudinal momentum deviation $\Delta p / p$ as a function of the longitudinal particle position $\Delta s$ with respect to the reference particle has certain characteristics directly after the linac. These are paramount for compression. In addition to a momentum deviation based on statistical uncertainties, the longitudinal phase space shows a correlated momentum deviation (chirp). This means that the average momentum deviation as a function of $\Delta s$ is not zero but depends on $\Delta s$ [see Fig. 3(a) for a typical tracked $3 \mathrm{nC}$ bunch and Fig. 3(b) for a $1 \mathrm{pC}$ bunch before the chicane]. In this paper the bunch length $\sigma_{s}$ is computed as the root mean square (rms) of the $\Delta s$-values. The rms momentum deviation $\sigma_{p}$ of a bunch is computed analogously. In fact, the Greek letter $\sigma$ will always indicate an rms quantity.

Since the momenta of particles at the head of the bunch lie below the momentum of the reference particle, the corresponding particles travel with a lower velocity compared to the tail of the bunch where the particle momenta lie above the momentum of the reference particle. The distributions in Fig. 3 were obtained by simulating the development of electron bunches from their generation at the cathode to the linac exit with the help of Astra. These are the bunches that we intend to use in the framework of the paper. Note that the typical length scale of a $3 \mathrm{nC}$ bunch 


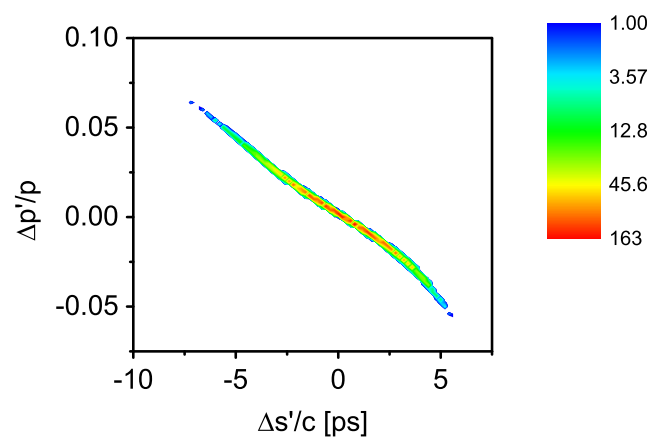

(a)

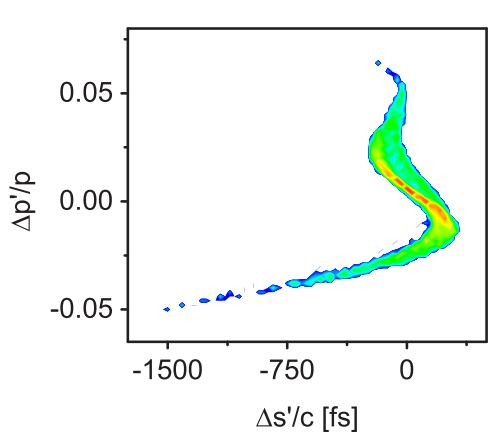

(c)

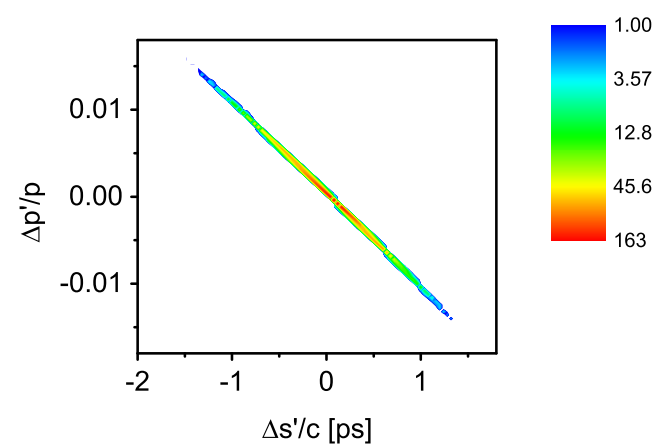

(b)

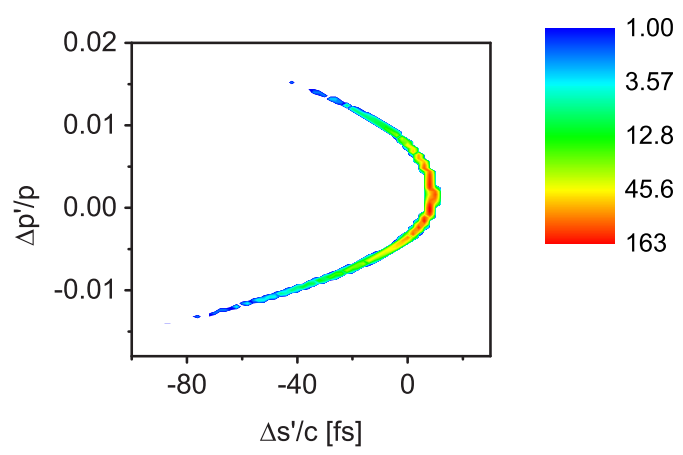

(d)

FIG. 3. Longitudinal phase space plots of $3 \mathrm{nC}$ and $1 \mathrm{pC}$ bunches (obtained in simulations) at the position $z=8.19 \mathrm{~m}$ before the chicane (a), (b) and at $z=12.65 \mathrm{~m}$ behind the chicane (c), (d). Here the normalized momentum deviation is plotted against the distance $\Delta s^{\prime}$ of a bunch particle with respect to the bunch center corresponding to the mean of all distances. The spatial bunch coordinates are divided by the speed of light to convert them to the dimension of time. Both distributions are centered on the mean relative momentum deviation at the vertical axis as well. (This procedure is conducted for all such distributions.) The rainbow color code represents the number of particles ranging from one (blue) to the maximum (red). The substructures for the $3 \mathrm{nC}$ bunch, i.e., the two small superimposed bumps originate from the emission of the particles at the cathode. The bunch parameters are $Q_{b}=3 \mathrm{nC}, \sigma_{s}=2.30 \mathrm{ps}$, $\sigma_{p}=1.87 \times 10^{-2}$ (a), $Q_{b}=1 \mathrm{pC}, \sigma_{s}=452 \mathrm{fs}, \sigma_{p}=4.79 \times 10^{-3}$ (b), $Q_{b}=3 \mathrm{nC}, \sigma_{s}=211 \mathrm{fs}(\mathrm{c})$, and $Q_{b}=1 \mathrm{pC}, \sigma_{s}=12.9 \mathrm{fs}(\mathrm{d})$.

directly before the FLUTE chicane lies in the picosecond regime, whereas the length of the $1 \mathrm{pC}$ bunch is several hundred femtoseconds.

Now we are interested in the longitudinal phase space after the chicane for the $3 \mathrm{nC}$ and the $1 \mathrm{pC}$ bunches used previously. Sending each electron along its own trajectory leads to the results shown in Figs. 3(c) and 3(d), respectively. Note that the units used for the horizontal axis are now femtoseconds. The rms bunch length was reduced by a factor of 10.9 for the $3 \mathrm{nC}$ bunch and a factor of 34.9 for the $1 \mathrm{pC}$ bunch. The double-s structure visible in Fig. 3(c) results from the superimposed bumps in the initial distribution shown in Fig. 3(a). Since all particle positions are reduced by compression this structure is now more evident than it had been in the latter figure.

Both the final bunch lengths and the bunch profiles of the analytical calculation in Figs. 3(c) and 3(d) agree well with Astra simulation results. For the $3 \mathrm{nC}$ bunch there is a deviation of the final bunch length of approximately $0.9 \%$ and for $1 \mathrm{pC}$ it is $13 \%$, which corresponds to around $2 \mathrm{fs}$ for both cases in absolute numbers. This is because dipole fringe fields are neglected in the analytical method, whereas in Astra they are described by a simple analytically defined dependence on distance.

\section{B. Sector chicane as a (hypothetical) example}

It is planned to construct the FLUTE bunch compressor using rectangular dipole magnets. However for theoretical reasons, in this paper we additionally intend to consider the characteristics of a bunch compressor made up of sector dipole magnets. A sector dipole is characterized by the property that the reference particle both enters and exits the magnet perpendicularly to its edges. This is not necessarily the case for a rectangular magnet.

The principle of a chicane constructed with sector dipole magnets is shown in Appendix A 1. The free parameters of such a chicane are the bending angle $\alpha$, the bending radius $R$, and the distances $L_{\text {space }}$ and $L_{\text {drift }}$. We can then derive a parametric representation of the reference trajectory. The result can be found in Appendix A 1 as well. Using this representation we compute the path length difference of two trajectories with normalized momentum deviation $\delta=\Delta p / p$. At first order in $\delta$ and for bending angles $\alpha \ll \pi / 2$ we obtain 


$$
\Delta L=-2 \alpha^{2}\left(\frac{2}{3} R \alpha+L_{\text {space }}\right) \delta+\mathcal{O}\left(\alpha^{4}, \delta^{2}\right)
$$

If the chicane parameters $\alpha, R$, and $L_{\text {space }}$ are chosen such that they correspond to the parameters of the chicane in Fig. 2, the momentum compaction factor for $\delta \ll 1$ and $\alpha \ll \pi / 2$ is the same for both types of chicanes. However, note that effects from the fringes of the dipole magnets have been neglected in this derivation. We will come back to the sector chicane at a later stage of the paper.

\section{TRANSFER MATRIX FORMALISM APPLIED ON THE FLUTE CHICANE}

In the previous chapter the FLUTE bunch compressor was investigated analytically by deriving parametric representations for particle trajectories in the compressor. The advantage of this approach is that all geometrical effects are taken into account. However this technique also has a number of disadvantages. First of all, the dipole field strength of the chicane magnets has been assumed to fall off to zero directly outside the magnet, i.e., we have used a hard-edge model. This is not the case for real magnets having a nonzero fringe field outside of the iron yoke. Second, the calculational time of this method is rather large since the trajectory for each electron has to be computed separately. This may already take several minutes for 5000 particles, which is the typical number of particles that we use.

For these reasons in the current section we are interested in applying the transfer matrix formalism. In general, each electron within a bunch can be described by a sixdimensional phase space vector $Z$, which reads as follows:

$$
\begin{aligned}
Z & =\left(\Delta x, \frac{p_{x}}{p}, \Delta y, \frac{p_{y}}{p}, \Delta s, \delta\right)^{T} \\
& \simeq\left(\Delta x, x^{\prime}, \Delta y, y^{\prime}, \Delta s, \delta\right)^{T}
\end{aligned}
$$

These components give positions in configuration space and momentum space with respect to a reference particle. The variables $\Delta x$ and $\Delta y$ are the two transverse offsets, $x^{\prime}$ and $y^{\prime}$ are the transverse angles, $\Delta s$ is the longitudinal position, and $\delta=\Delta p / p$ the normalized momentum deviation.

In the framework of perturbation theory the equation of motion for an electron is not solved exactly, but as an expansion in the deviations $\Delta x, x^{\prime}$ etc. from the reference trajectory. Each part of an accelerator transforms an initial phase space vector $Z^{(1)}$ to a final vector $Z^{(2)}$. Expanding this transformation to second order in the phase space vector it can be written with the help of a transfer matrix $R$ (a second-rank tensor) and a third-rank transfer tensor $T$ $[18,19]$ :
$Z_{j}^{(2)}=Z_{j}^{(1)}+\sum_{k=1}^{6} R_{j k} Z_{k}^{(1)}+\sum_{k, l=1}^{6} T_{j k l} Z_{k}^{(1)} Z_{l}^{(1)}+\cdots$

If an electron propagates through an element designated by (a) and followed by an element (b) the resulting transfer matrix is given by $R^{c}=R^{b} R^{a}$. The third-rank tensor of a combination of two accelerator components (a) and (b) is given by [19]

$$
T_{i j k}^{c}=\sum_{l=1}^{6} R_{i l}^{b} T_{l j k}^{a}+\sum_{l=1}^{6} \sum_{m=1}^{6} T_{i l m}^{b} R_{l j}^{a} R_{m k}^{a} .
$$

This equation involves both the transfer matrices and the third-rank tensors of the corresponding accelerator components.

The chicane transforms the chirp of the bunch. Restricting ourselves to the two-dimensional longitudinal phase space spanned by $(\Delta s, \delta)$ leads to the following (well-known) transformation at linear order:

$$
\begin{aligned}
& Z^{(2)}=R Z^{(1)}, \quad Z^{(1)} \equiv\left(\begin{array}{c}
\Delta s^{(1)} \\
\delta
\end{array}\right), \\
& Z^{(2)} \equiv\left(\begin{array}{c}
\Delta s^{(2)} \\
\delta
\end{array}\right), \quad R=\left(\begin{array}{cc}
1 & R_{56} \\
0 & 1
\end{array}\right), \\
& R_{56}=2\left(\frac{L_{\mathrm{mag}}}{R}\right)^{2}\left(\frac{2}{3} L_{\mathrm{mag}}+L_{\text {space }}\right) .
\end{aligned}
$$

Herein $\Delta s^{(1)}$ and $\Delta s^{(2)}$ are the longitudinal positions of a bunch particle before and after compression, respectively. The momentum compaction factor $R_{56}>0$ relates the longitudinal position to the normalized momentum deviation. The expression stated for $R_{56}$ holds for $L_{\text {mag }} \ll R$. It can be checked that for the total transfer matrix of the chicane, $R_{51}$ and $R_{52}$ are approximately zero in the same limit. This explains why initial transverse offsets and angle deviations have a small impact on the final bunch length producing only effects at higher order.

\section{A. First-order perturbation theory}

First of all, we will concentrate simply on the transfer matrices $R$ that are taken from [19]. The notation used in $[18,19]$ will be kept with some minor modifications that will be stated in the corresponding context. For the FLUTE chicane the transfer matrices of a drift and that of a rectangular dipole are needed. For a drifting particle both $\Delta x$ and $\Delta y$ increase with the length of the drift whereas the transverse angles $x^{\prime}$ and $y^{\prime}$ are not modified. Since a particle travels on parts of a circle through a sector magnet, the corresponding transfer matrix involves trigonometric functions. If the particle does not enter or exit the dipole magnet perpendicularly to its surfaces, magnetic fringe fields have 
to be taken into account. The drift transfer matrix involves the entrance and exit angles of the particle with respect to the magnet edges.

The bunch profiles and final bunch lengths obtained by first-order perturbation theory differ from the Astra results by quite some amount. For the $3 \mathrm{nC}$ bunch the deviation is $14 \%$ and for the $1 \mathrm{pC}$ bunch it is even $43 \%$. This shows that the transfer matrix formalism at first order in the momentum deviation does not suffice to reproduce the Astra simulation output.

\section{B. Second-order corrections}

The previous section dealt with the momentum compaction factor at first-order perturbation theory. We are now interested to compute the second-order contribution of the path length difference in the chicane, i.e., the contribution proportional to $\delta^{2}$. It is given by the tensor coefficient $T_{566}$ and can be obtained from Eqs. (2.2) and (2.3) by including terms in the Taylor expansion up to second order in $\delta$. For $L_{\text {mag }} \ll R$ it reads

$$
\begin{aligned}
\Delta L \equiv & \Delta L^{(1)}+\Delta L^{(2)}+\cdots \\
= & -2\left(\frac{L_{\mathrm{mag}}}{R}\right)^{2}\left[\frac{2}{3} L_{\mathrm{mag}}+L_{\text {space }}\right] \delta \\
& +\left(\frac{L_{\mathrm{mag}}}{R}\right)^{2}\left[2 L_{\mathrm{mag}}+3 L_{\text {space }}\right] \delta^{2}+\cdots,
\end{aligned}
$$

where $\Delta L^{(n)}$ denotes a correction proportional to $\delta^{n}$. From the general relation $\Delta L=R_{56} \delta+T_{566} \delta^{2}+\cdots$, the coefficient $T_{566}$ can be directly obtained by comparison:

$$
T_{566}=\left(\frac{L_{\mathrm{mag}}}{R}\right)^{2}\left[2 L_{\mathrm{mag}}+3 L_{\mathrm{space}}\right]=-\frac{3}{2} R_{56} .
$$

Note that $T_{566}$ has the same order of magnitude as $R_{56}$ but it has a different sign. These results match with what was obtained in [20].

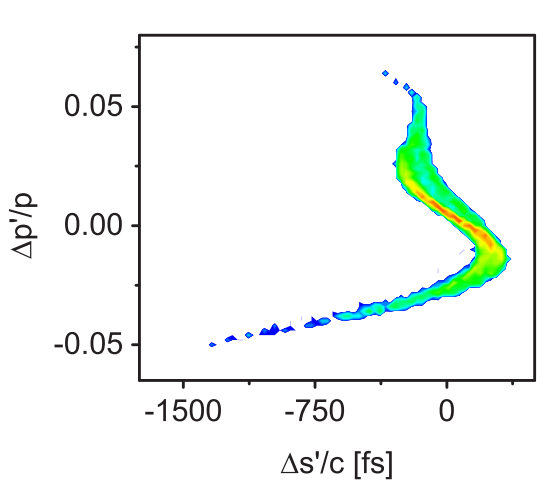

(a)
Now let us compare the bunch profiles obtained from the transfer matrix formalism at second order in $\Delta p / p$ to the Astra simulation output. In Figs. 4(a) and 4(b) the bunch profiles for the bunch charges $3 \mathrm{nC}$ and $1 \mathrm{pC}$, respectively, are shown. The rms bunch length for $3 \mathrm{nC}$ is approximately $7 \%$ larger than the Astra result whereas the bunch length for $1 \mathrm{pC}$ is $10 \%$ smaller. In comparison to perturbation theory at first order in $\Delta p / p$ the final bunch profile at second order agrees much better with the simulations. The first-order contribution has the trend to underrate the final bunch length. This is corrected by the additional $T_{566}$ contribution having the opposite sign as the $R_{56}$ term.

In this context we intend to compute $T_{566}$ of the chicane by using Eq. (3.3). The latter equation decomposes into the first-order and second-order transfer coefficients of the individual components of the chicane such as the bending magnets and drifts. However not all of the needed coefficients, which relate path length differences to differences in angles and the momentum deviation, can be found in the literature. At first we performed a computation according to Eq. (3.3) showing that the result of Eq. (3.6) cannot be obtained with the sets of coefficients listed in, e.g., $[18,19]$. For this reason we conclude that the sets of coefficients relating the path length difference to the five remaining phase space variables are not complete. Hence the goal is to derive the missing ones as follows.

For the derivation consider Fig. 5, which shows the particle trajectories in the first two dipole magnets of a bunch compressor. Both the FLUTE chicane consisting of rectangular dipole magnets and a hypothetical chicane of sector dipole magnets is considered. The regions where path length differences at second order in $\delta$ occur are encircled. The method is to extract the relevant coefficients from the trajectories, i.e., from the solutions of the equations of motion. It can deliver results quite fast provided that the solution is on hand, which is the case here. Note that if the exact solutions are not available the technique of Lie

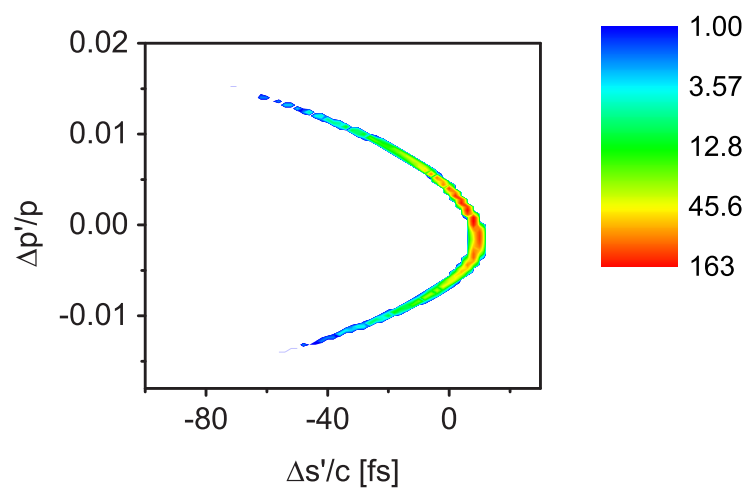

(b)

FIG. 4. Longitudinal phase space plots of $3 \mathrm{nC}$ and $1 \mathrm{pC}$ bunches (obtained in simulations) after the chicane. The profiles shown were computed by using the transfer matrix formalism at second order in $\Delta p / p$ using $R_{56}$ of Eq. (3.4) and $T_{566}$ of Eq. (3.6). The bunch parameters are $Q_{b}=3 \mathrm{nC}, \sigma_{s}=214 \mathrm{fs}$ (a) and $Q_{b}=1 \mathrm{pC}, \sigma_{s}=11 \mathrm{fs}(\mathrm{b})$. 


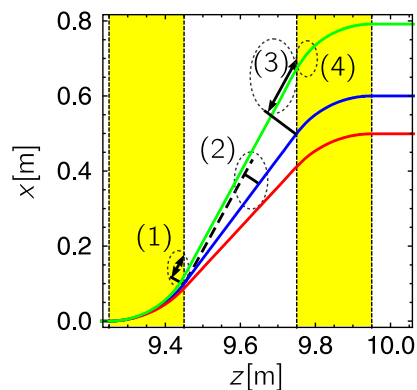

(a)

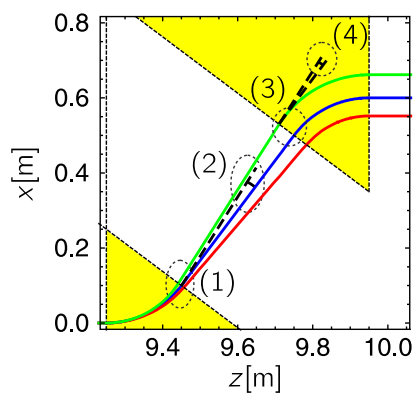

(b)
FIG. 5. Particle trajectories inside the first two chicane magnets. The reference trajectory for a particle momentum of $p=$ $40.66 \mathrm{MeV}$ is shown in blue. The green trajectory is that for a particle with lower momentum $p+\Delta p=(1-0.09) p$. The particle traveling along the red trajectory has a higher momentum $p+\Delta p=(1+0.09) p$. The left panel (a) shows the trajectories in a chicane consisting of rectangular dipole magnets with $L_{\text {mag }}=0.2 \mathrm{~m}, L_{\text {space }}=0.3 \mathrm{~m}, L_{\text {drift }}=1.0 \mathrm{~m}$ and the hypothetical bending radius $R=0.25 \mathrm{~m}$. The latter exaggerated value has been chosen such that the difference in the path lengths becomes visible. The right panel (b) shows the trajectories in a chicane of sector bending magnets. Here the chicane parameters are chosen such that the path length of the reference trajectory is equal to the path length of the corresponding trajectory in (a). The regions where path length differences proportional to $\delta^{2}$ originate from are encircled and marked by (1), (2), (3), and (4). The yellow areas show the dipole magnets.

algebraic maps is more suitable $[19,21]$. However, we will not follow the latter approach in this paper.

The following two sections are rather technical. Readers who are only interested in the results may skip them and look at Table I where the results for rectangular dipole magnets are summarized.

\section{Rectangular dipole magnets}

First we consider the rectangular D-shape bunch compressor that is planned for FLUTE [see Fig. 5(a)]. This chicane has a mirror symmetry with respect to an axis that is parallel to one of the transverse axes and has a distance
$2 L_{\text {mag }}+L_{\text {space }}+L_{\text {drift }} / 2$ from the left edge of the first magnet. The path length difference of the chicane from its start to the symmetry axis mentioned is $1 / 2$ of the result given by Eq. (3.5). Therefore it is sufficient to consider only the first two magnets. By doing so, we are interested in the origin of the terms that make up $\Delta L^{(2)}$. The comparison of terms is understood to be based on the assumptions $\beta=$ $v / c=1$ and $\alpha \ll \pi / 2$, which will not be mentioned for every instance.

(1) The first difference in path lengths at order $\delta^{2}$ comes from region (1) in Fig. 5(a), i.e., it occurs in the vicinity of the exit face of the first dipole magnet. Computing the difference in path length within the magnet as a function of $\delta$ results in

$$
\Delta L_{\mathrm{rec}}=R(\alpha-\tan \alpha) \delta+\frac{R}{2}\left(\tan ^{3} \alpha\right) \delta^{2}+\mathcal{O}\left(\delta^{3}\right)
$$

Note that the first-order term in $\delta$ corresponds to the element $R_{56}$ of the sector dipole matrix with $n=0$ (neglecting magnetic field inhomogeneities). The only difference is the occurrence of $\tan \alpha$ instead of $\sin \alpha$. However both functions coincide for bending angles $\alpha \ll \pi / 2$, which is the case for the FLUTE chicane. The second term of Eq. (3.7) then leads to

$$
\begin{aligned}
\Delta L_{1}^{(2)} & =\Delta L_{\mathrm{rec}}^{(2)} \\
& =\frac{1}{2}\left(\frac{L_{\mathrm{mag}}}{R}\right)^{2} L_{\mathrm{mag}} \delta^{2}+\mathcal{O}\left[\left(L_{\mathrm{mag}} / R\right)^{4}\right] .
\end{aligned}
$$

Performing the analogue computation for a sector magnet we obtain the following result for the path length difference:

$$
\Delta L_{\mathrm{sec}}=R(\alpha-\sin \alpha) \delta-\frac{R}{6}\left(\sin ^{3} \alpha\right) \delta^{3}+\mathcal{O}\left(\delta^{4}\right)
$$

Contrary to Eq. (3.7) there is no term proportional to $\delta^{2}$. Therefore the path length difference at second order in $\delta$ in Eq. (3.7) is not related to the body of the magnet. That is

TABLE I. Path length differences at second order in $\delta$ for the chicane consisting of rectangular dipole magnets. The first two columns show the contribution to the path length difference. The third column presents how each contribution can be expressed via the transfer matrix and third-rank tensor coefficients. The last two columns list the individual matrix and tensor coefficients plus their specific values.

\begin{tabular}{lcccc}
\hline \hline Contribution & & Composition & Coefficient & Value \\
\hline$\Delta L_{1}^{(2)}$ & $L_{\text {mag }}^{2} /(2 R)$ & $T_{56}^{\text {exi fringe }}$ & $T_{566}^{\text {exit fringe }}$ & $\tan ^{2}(\alpha) /(2 h)$ \\
$\Delta L_{2}^{(2)}$ & $L_{\text {mag }}^{2} L_{\text {space }} /(2 R)$ & $T_{522}^{\text {drift }}\left(R_{26}^{\text {exit fringe }}\right)^{2}$ & $T_{522}^{\text {drift }}$ & $L /(2 \beta)$ \\
& & & $R_{26}^{\text {exit fringe }}$ & $\tan \alpha$ \\
$\Delta L_{3}^{(2)}$ & $L_{\text {mag }}^{2} L_{\text {space }} / R$ & $\left(R_{52}^{\text {entr. fringe }}\right)\left(T_{266}^{\text {exit fringe }}\right)$ & $R_{52}^{\text {entr. fringe }}$ & $L \tan \alpha$ \\
& & & $T_{266}^{\text {exit fringe }}$ & $-\tan \alpha$ \\
$\Delta L_{4}^{(2)}$ & $L_{\text {mag }}^{3} /(2 R)$ & $T_{566}^{\text {exit fringe }}$ & $T_{566}^{\text {exit fringe }}$ & $\tan ^{3}(\alpha) /(2 h)$ \\
\hline \hline
\end{tabular}


why the magnet fringe must deliver a contribution to the path length difference proportional to $\delta^{2}$. This is described by a tensor coefficient $T_{566}$ whose value can be obtained from Eq. (3.7):

$$
T_{566}^{\text {exit fringe }}=\frac{1}{2 h} \tan ^{3} \alpha .
$$

Such a coefficient should be taken into account for the exit fringe of a rectangular dipole magnet with curvature $h=$ $1 / R$ and bending angle $\alpha$.

(2) The path length difference in the region between the first and second dipole magnet has two main contributions at second order in $\delta$. The origin of the first contribution is given by region (2) in Fig. 5(a). It is related to the exit angle of the first dipole magnet with respect to the reference trajectory as a function of $\delta$. The latter results from the scalar product of the corresponding tangent vectors $\hat{\mathbf{t}}$ of the trajectories at the magnet exit:

$$
\begin{aligned}
\Delta \phi & =\arccos \{\hat{\mathbf{t}}[R+\Delta R, \alpha(R+\Delta R)] \cdot \hat{\mathbf{t}}[R, \alpha(R)]\} \\
& =(\tan \alpha) \delta-\tan \alpha\left(\frac{3+\cos (2 \alpha)}{4 \cos ^{2} \alpha}\right) \delta^{2}+\mathcal{O}\left(\delta^{3}\right) .
\end{aligned}
$$

From Eq. (3.11) we can read off the following transfer matrix and third-rank tensor coefficients that relate $\Delta \phi$ to the momentum deviation and to its square, respectively:

$$
\begin{gathered}
R_{26}^{\text {exit fringe }}=\tan \alpha, \\
T_{266}^{\text {exit fringe }}=-\tan \alpha\left(\frac{3+\cos (2 \alpha)}{4 \cos ^{2} \alpha}\right) \\
=-\tan \alpha+\mathcal{O}\left(\alpha^{3}\right) .
\end{gathered}
$$

At the exit of the first dipole magnet the momentum deviation $\delta$ is translated to an angle $\Delta \phi$ via Eq. (3.11). This is a contribution at first-order perturbation theory in $\delta$. The path length difference between two drifts that enclose an angle $\Delta \phi$ is of second order in this angle. That is why the aforementioned $\Delta \phi$ then leads to a second-order path length difference in the drift behind the first dipole magnet. Using the first term of Eq. (3.11) we obtain

$$
\begin{aligned}
\Delta L_{2}^{(2)} & =\frac{L_{\text {space }}}{\cos (\alpha+\Delta \phi)}-\frac{L_{\text {space }}}{\cos \alpha} \\
& =\frac{\left(\Delta \phi^{(1)}\right)^{2}}{2} \frac{L_{\text {space }}}{\cos \alpha}\left(1+2 \tan ^{2} \alpha\right)+\mathcal{O}\left(\delta^{3}\right) \\
& =\frac{1}{2}\left(\frac{L_{\text {mag }}}{R}\right)^{2} L_{\text {space }} \delta^{2}+\mathcal{O}\left[\left(L_{\text {mag }} / R\right)^{4} \delta^{2}, \delta^{3}\right] .
\end{aligned}
$$

The latter equation relates the path length difference of a drift to the square of an angle with respect to the reference particle. This is why it will be described by a product $T_{522} R_{26}^{2}$ where $R_{26}$ is given by Eq. (3.12a). The coefficient
$T_{522}$ must be that of a drift but these are not listed in $[18,19]$. However they are contained in the MAD-X Fortran programming code [22] and are given by

$T_{126}^{\mathrm{drift}}=\frac{L}{2 \beta}=T_{162}^{\mathrm{drift}}=T_{346}^{\mathrm{drift}}=T_{364}^{\mathrm{drift}}=T_{522}^{\mathrm{drift}}=T_{544}^{\mathrm{drift}}$,

with $\beta=v / c$ and the length $L$ of the drift space. We see that for $L=L_{\text {space }}$ the product $T_{522}^{\text {drift }}\left(R_{26}^{\text {exit fringe }}\right)^{2}$ corresponds to Eq. (3.13).

(3) The second contribution for path length differences proportional to $\delta^{2}$ in the drift space behind the first dipole magnet is related to region (3) in Fig. 5(a). A trajectory enclosing an angle $\Delta \phi$ with the reference trajectory has an additional length $\Delta L$ within the drift space because the trajectory encloses a nonzero angle with the entrance edge of the second dipole magnet. With the second-order term in Eq. (3.11) we obtain a second-order correction to the path length difference with respect to the momentum deviation $\delta$ :

$$
\begin{aligned}
\Delta L_{3}^{(2)} & =\frac{L_{\text {space }}}{\cos (\alpha+\Delta \phi)}-\frac{L_{\text {space }}}{\cos \alpha} \\
& =\frac{L_{\text {space }}}{\cos \alpha} \tan \alpha\left|\Delta \phi^{(2)}\right|+\mathcal{O}\left(\delta^{3}\right) \\
& =\frac{L_{\text {space }}}{\cos \alpha}\left(\frac{3+\cos (2 \alpha)}{4 \cos ^{2} \alpha}\right)(\tan \alpha)^{2} \delta^{2}+\mathcal{O}\left(\delta^{3}\right) \\
& =\left(\frac{L_{\text {mag }}}{R}\right)^{2} L_{\text {space }} \delta^{2}+\mathcal{O}\left[\left(L_{\text {mag }} / R\right)^{4} \delta^{2}, \delta^{3}\right] \\
& =2 \Delta L_{2}^{(2)}+\mathcal{O}\left[\left(L_{\mathrm{mag}} / R\right)^{4} \delta^{2}, \delta^{3}\right] .
\end{aligned}
$$

Since Eq. (3.15) involves a second-order angle the coefficient responsible for this path length contribution must be of first order, i.e., an $R_{52}$. As it is related to the fringe of a rectangular dipole magnet we obtain

$$
\Delta L=(L \tan \alpha) \Delta \phi \Rightarrow R_{52}^{\text {entr. fringe }}=L \tan \alpha,
$$

where $L$ is the length of the drift space before the corresponding dipole magnet. With $L=L_{\text {space }}$ the product $\left(R_{52}^{\text {entr. fringe }}\right)\left(T_{266}^{\text {exit fringe }}\right)$ is equal to the result of Eq. (3.15). The sign of the angle in $T_{266}^{\text {exit fringe }}$ of Eq. (3.12) has to be chosen as negative in the first magnet leading to the correct overall sign.

(4) Finally, we end up with region (4) in Fig. 5(a) leading to a second-order correction that corresponds to the correction of region (1):

$$
\begin{aligned}
\Delta L_{4}^{(2)} & =\Delta L_{1}^{(2)} \\
& =\frac{1}{2}\left(\frac{L_{\mathrm{mag}}}{R}\right)^{2} L_{\mathrm{mag}} \delta^{2}+\mathcal{O}\left[\left(L_{\mathrm{mag}} / R\right)^{4}\right] .
\end{aligned}
$$


Here it is related to the entrance fringe of the second dipole magnet. So it can only come from

$$
T_{566}^{\text {exit fringe }}=\frac{1}{2 h} \tan ^{3} \alpha .
$$

Summing up $\Delta L_{i}^{(2)}$ for $i=1 \cdots 4$ and multiplying the result by 2 leads to $\Delta L^{(2)}$ of Eq. (3.5).

\section{Sector dipole magnets}

In the current section we are interested in the path length difference at second order in $\delta$ for the hypothetical bunch compressor made up of sector dipole magnets (see Sec. II B). This further example will be studied for academic reasons to understand the differences to the D-shape chicane of rectangular magnets. The path length difference at first and second order in $\delta$ is given by

$$
\begin{aligned}
\Delta L= & -2 \alpha^{2}\left(\frac{2}{3} R \alpha+L_{\text {space }}\right) \delta+\alpha^{2}\left(2 R \alpha+3 L_{\text {space }}\right) \delta^{2} \\
& +\mathcal{O}\left(\alpha^{4}, \delta^{3}\right) .
\end{aligned}
$$

From the previous equation we can extract the third-rank tensor element for this chicane relating the momentum deviation to the path length difference:

$$
T_{566}=\alpha^{2}\left(2 R \alpha+3 L_{\text {space }}\right)=-\frac{3}{2} R_{56}
$$

We see that this is connected to the matrix element $R_{56}$ in the same manner as for the chicane of rectangular magnets. Analogous to Sec. III B 1 we now intend to derive the thirdrank tensor coefficients for sector dipole magnets such that this result can be reproduced.

For the sector chicane we were also able to identify four regions where path length differences originate from [see Fig. 5(b)]. As we saw in Eq. (3.9) there is no path length difference $\Delta L$ in a sector dipole magnet at second order in the normalized momentum deviation $\delta$. The major part of $\Delta L^{(2)}$ emerges at the second dipole magnet. Because of transverse displacements $\Delta x$, which emerge at several places, a particle travels an approximate path length $(R+\Delta x) \alpha$ resulting in $\Delta L=\alpha \Delta x$.

(1) The first angular displacement $\Delta x_{1}$ already appears at the exit fringe of the first dipole magnet, i.e., at region (1) in Fig. 5(b). It is given by

$$
\Delta x_{1}=2 R \sin ^{2}\left(\frac{\alpha}{2}\right) \delta+\frac{1}{2}\left(R \sin ^{2} \alpha\right) \delta^{2}+\mathcal{O}\left(\delta^{3}\right) .
$$

As indicated, this displacement leads to a longer path length in the second dipole magnet. Its contribution at second order in $\delta$ is

$$
\begin{aligned}
\Delta L_{1}^{(2)} & =\Delta x_{1}^{(2)} \alpha=\frac{1}{2} R \alpha \sin ^{2} \alpha \delta^{2} \\
& =\frac{1}{2} R \alpha^{3} \delta^{2}+\mathcal{O}\left(\alpha^{5}\right) .
\end{aligned}
$$

From Eq. (3.21) we extract the transfer coefficients relating the first transverse coordinate with the momentum deviation:

$$
\begin{aligned}
R_{16}^{\mathrm{sec}} & =2 R \sin ^{2}\left(\frac{\alpha}{2}\right)=R(1-\cos \alpha), \\
T_{166}^{\mathrm{sec}} & =\frac{1}{2}\left(R \sin ^{2} \alpha\right) .
\end{aligned}
$$

Neglecting magnetic field inhomogeneities we obtain from the transfer matrix of the sector dipole that $R_{51}^{\mathrm{sec}}=$ $-\sin (\alpha) / \beta$ with $\beta=v / c$. For $\alpha \ll \pi / 2$ and $\beta=1$ the product $\left(R_{51}^{\mathrm{sec}}\right)\left(T_{166}^{\mathrm{sec}}\right)$ corresponds to the result of Eq. (3.22).

(2) Any particle with normalized momentum deviation $\delta$ exits the first dipole magnet with an angle $\Delta \phi$ with respect to the reference particle:

$$
\begin{aligned}
\Delta \phi= & (\sin \alpha) \delta+(\sin \alpha) \delta^{2} \\
& +\frac{1}{12}[13-\cos (2 \alpha)](\sin \alpha) \delta^{3}+\mathcal{O}\left(\delta^{4}\right) .
\end{aligned}
$$

From the latter equation we obtain

$$
R_{26}^{\mathrm{sec}}=\sin \alpha, \quad T_{266}^{\mathrm{sec}}=\sin \alpha .
$$

There is one contribution to $\Delta L$ at second order in $\delta$ that coincides with $\Delta L_{2}^{(2)}$ obtained for the rectangular dipole magnet. Consider region (2) in the drift space between the first two magnets. A particle propagating along a trajectory that encloses an angle $\Delta \phi$ with the reference trajectory travels a different path length at second order in $\delta$. It involves the first-order contribution of the angle $\Delta \phi$ of Eq. (3.24):

$$
\begin{aligned}
\Delta L_{2}^{(2)} & =\frac{L_{\text {space }}}{\cos (\alpha+\Delta \phi)}-\frac{L_{\text {space }}}{\cos \alpha} \\
& =\frac{\left(\Delta \phi^{(1)}\right)^{2}}{2} \frac{L_{\text {space }}}{\cos \alpha}\left(1+2 \tan ^{2} \alpha\right)+\mathcal{O}\left(\delta^{3}\right) \\
& =\frac{\left(1+2 \tan ^{2} \alpha\right) \sin ^{2} \alpha}{2 \cos \alpha} L_{\text {space }} \delta^{2}+\mathcal{O}\left(\delta^{3}\right) \\
& =\frac{1}{2} \alpha^{2} L_{\text {space }} \delta^{2}+\mathcal{O}\left(\delta^{3}, \alpha^{4}\right) .
\end{aligned}
$$

Using $T_{522}^{\text {drift }}=L_{\text {space }} /(2 \beta)$ of Eq. (3.14) the product $T_{522}^{\text {drift }}\left(R_{26}^{\text {sec }}\right)^{2}$ equals Eq. (3.26).

(3) Due to the second-order contribution of $\Delta \phi$ the drift space between the first two magnets leads to a further transverse displacement at the entrance of the second dipole 
magnet. This corresponds to region (3) in Fig. 5(b) and the displacement reads

$$
\begin{aligned}
\Delta x_{2} & =\frac{L_{\text {space }}}{\cos \alpha} \tan \Delta \phi=\frac{L_{\text {space }}}{\cos \alpha} \Delta \phi^{(2)}+\mathcal{O}\left(\delta^{3}\right) \\
& =\frac{L_{\text {space }}}{\cos \alpha} \sin \alpha \delta^{2}+\mathcal{O}\left(\delta^{3}\right) .
\end{aligned}
$$

It again translates to a path length difference at second order in $\delta$ analogous to Eq. (3.22):

$$
\begin{aligned}
\Delta L_{3}^{(2)} & =\Delta x_{2}^{(2)} \alpha=\frac{\alpha \sin \alpha}{\cos \alpha} L_{\text {space }} \delta^{2} \\
& =\alpha^{2} L_{\text {space }} \delta^{2}+\mathcal{O}\left(\alpha^{4}\right)
\end{aligned}
$$

Computing the product $\left(R_{51}^{\mathrm{sec}}\right)\left(R_{12}^{\mathrm{drift}}\right)\left(T_{266}^{\mathrm{sec}}\right)$ with $R_{51}^{\mathrm{sec}}=$ $-\sin (\alpha) / \beta, R_{12}^{\text {drift }}=L_{\text {drift }}$ (see the drift matrix in [19]), and $T_{266}^{\mathrm{sec}}$ of Eq. (3.25) results in $\Delta L_{3}^{(2)}$.

(4) The fourth contribution to the whole $\Delta L$ proportional to $\delta^{2}$ comes from the fact that a particle enters the second magnet under the angle $\Delta \phi$ with respect to the reference particle. That is marked as region (4) in Fig. 5(b).

$$
\begin{aligned}
\Delta L_{4}^{(2)} & =R(1-\cos \alpha) \Delta \phi^{(2)}=R(1-\cos \alpha) \sin \alpha \delta^{2} \\
& =\frac{1}{2} R \alpha^{3} \delta^{2}+\mathcal{O}\left(\alpha^{5}\right) .
\end{aligned}
$$

This result agrees with $\left(R_{52}^{\mathrm{sec}}\right)\left(T_{266}^{\mathrm{sec}}\right)$ where $T_{266}^{\mathrm{sec}}$ is taken from Eq. (3.25). The matrix element $R_{52}^{\mathrm{sec}}=-R(1-\cos \alpha) / \beta$ is obtained from the transfer matrix of the sector dipole, again neglecting field inhomogeneities.

Summing up $\Delta L_{i}^{(2)}$ for $i=1 \cdots 4$ and multiplying the result by 2 leads to the second-order term in Eq. (3.19).

The results obtained are summarized in Table I. We have demonstrated that the second-order coefficient $T_{566}$ of the total chicane, Eq. (3.6), can be obtained by using Eq. (3.3) with the new coefficients found. The individual contributions $\Delta L_{i}^{(2)}$ were tracked back to specific magnetic fringes, angles with respect to the reference trajectory or transverse displacements. These terms are made up of third-rank tensor coefficients or products of transfer matrix elements with tensor coefficients. Each of them must have a structure "566" of free indices relating the momentum deviation square to a path length difference. The procedure employed demonstrates how hitherto unknown second-order transfer coefficients of accelerator components being part of a bunch compressor can be obtained directly by the following recipe: (1) Set up the exact particle trajectory piecewisely from the solutions of the equations of motion for each individual component. (2) Compute the path length as a function of the particle momentum and obtain $T_{566}$ of the total chicane from a Taylor expansion. (3) Use the composition formula of Eq. (3.3) to calculate $T_{566}$ of the chicane based on an available list of first- and second-order transfer coefficients. If both results match the list of coefficients is complete for this purpose. (4) If the results are different some coefficients may be missing. In this case track back possible path length differences to different entrance and exit angles of dipoles, different path lengths at dipole fringes, etc. (5) Extract missing transfer coefficients from the contributions found in the previous step.

By doing so, second-order coefficients can be computed without relying on advanced methods of, e.g., [19,21].

\section{SPACE CHARGE EFFECTS}

So far, the FLUTE bunch compressor has been considered merely from the geometrical point of view. We investigated how a bunch evolves when each particle is sent along its own trajectory through the chicane. The results agree well with what is obtained from Astra simulations with the space charge routine switched off. Furthermore the FLUTE chicane was examined with the transfer matrix formalism being a well-known tool in accelerator physics. Within this perturbative method the first order is not sufficient to reproduce the simulation results, but the second-order terms in the momentum deviation are necessary. In the analytical calculations performed so far, both space charge effects and the backreaction of CSR on the bunch were neglected.

The next step lies in taking space charge forces into account, i.e., the mutual interaction of bunch particles due to the attraction and repulsion by their electromagnetic fields. There are various tools available that treat space charge effects numerically, e.g., by solving the Poisson equation. Some examples are the already mentioned Astra [17], CSRtrack [23], and PARMELA [24]. The approach within this section is (semi)analytical and our goal is to provide a set of formulas to estimate the amount of increase in bunch length due to space charge forces. Our intention is to get insights into the physical processes responsible for bunch elongation. Thereby we follow the procedure described in the fourth chapter of [25]. This will be applied to both the $3 \mathrm{nC}$ and the $1 \mathrm{pC}$ bunches considered before. As a starting point, the influence of space charge forces on the bunch will be estimated by simple principles. Every charged particle beam can be considered as a plasma, i.e., as a gas of charged particles. The space charge forces acting on a particle moving in the transverse direction originate from the electric and magnetic fields. Assuming a uniform, cylindric particle distribution, these forces depend linearly on the transverse coordinate $x$ and they are related to what is known as the plasma frequency $\omega_{p}$. The latter is given by

$$
\omega_{p}=\sqrt{\frac{e^{2} n}{\varepsilon_{0} \gamma^{3} m}}, \quad n=\frac{Q_{b}}{e \pi \sigma_{x} \sigma_{y} \cdot\left(2 \sigma_{s}\right)},
$$

where $e$ is the elementary charge, $\varepsilon_{0}$ the vacuum permittivity, $m$ the electron mass, and $\gamma$ is the Lorentz factor of the 
bunch. Furthermore, $n$ is the number density of electrons, $Q_{b}$ the bunch charge, $\sigma_{i}$ for $i=(x, y)$ is the rms transverse beam size, and $\sigma_{s}$ the rms longitudinal bunch length. Note that for the cylinder length we use the double rms longitudinal bunch length $2 \sigma_{s}$ since $\sigma_{s}$ is the standard deviation from the mean and, therefore, it is a measure for one half of the width of the distribution. This procedure is employed for simplicity and a more detailed consideration will be provided in Sec. IVA.

Electrons in a plasma oscillate with the plasma frequency. While the plasma frequency describes a transverse oscillation it nevertheless involves the Lorentz factor. The reason is that the relativistic mass and the relativistic electric and magnetic fields go into the corresponding equation of motion. To get a feeling for the sizes of these values at FLUTE the $3 \mathrm{nC}$ and $1 \mathrm{pC}$ bunches from above will be considered, in particular. We are interested in the behavior of the bunches right before the fourth chicane magnet. ${ }^{1}$ Space charge effects are expected to be most important in this magnet as here the bunch has already been compressed by the largest fraction. The characteristic values of these bunches, e.g., the bunch length are obtained with the trajectory method. Hence, we assume that space charge effects are negligible before the fourth magnet. The results can be found in Table II and we then obtain

$$
\begin{gathered}
n=\left\{\begin{array}{l}
6.2 \times 10^{18} 1 / \mathrm{m}^{3} \text { for } 3 \mathrm{nC}, \\
4.3 \times 10^{17} 1 / \mathrm{m}^{3} \text { for } 1 \mathrm{pC},
\end{array}\right. \\
\omega_{p}= \begin{cases}1.9 \times 10^{8} 1 / \mathrm{s} & \text { for } 3 \mathrm{nC}, \\
5.1 \times 10^{7} 1 / \mathrm{s} & \text { for } 1 \mathrm{pC} .\end{cases}
\end{gathered}
$$

Although these frequencies seem to be very high, they are heavily suppressed by the Lorentz factor-contrary to a nonrelativistic plasma with these particle densities.

A characteristic quantity for the behavior of space charge forces in a particle beam is the Debye length $\lambda_{D}$ being the ratio of the rms transverse velocity $\tilde{v}_{x}$ and the plasma frequency:

$$
\lambda_{D}=\frac{\tilde{v}_{x}}{\omega_{p}}=\sqrt{\frac{\varepsilon_{0} \gamma^{2} k_{B} T}{e^{2} n}},
$$

with Boltzmann's constant $k_{B}$. The Debye length emerges as a length scale in the Poisson equation of a distribution of charged particles. It is a measure of the influence that each particle has on the other particles within a plasma. If the Debye length lies in the order of the beam dimensions, the smeared-out behavior of the particle distribution will be more important than the interaction of single particles. For a Debye length in the order of the distances between the

\footnotetext{
${ }^{1}$ We obtain the corresponding distribution with the trajectory method described in Sec. II. Thereby we assume that the change of transverse coordinates is negligible.
}

TABLE II. Physical parameters used for the $3 \mathrm{nC}$ and the $1 \mathrm{pC}$ bunches, respectively, before the fourth chicane magnet (at $z=11.45 \mathrm{~m}$ ). The momentum deviation $\Delta p$, the bunch length $L$, and the beam sizes $\sigma_{x}, \sigma_{y}$ are rms values. The transverse velocities $\tilde{v}_{x}, \tilde{v}_{y}$ are defined as the velocities corresponding to the rms values of the transverse momentum components $p_{x}$ and $p_{y}$, respectively.

\begin{tabular}{lccc}
\hline \hline Parameter & Unit & $Q_{b}=3 \mathrm{nC}$ & $Q_{b}=1 \mathrm{pC}$ \\
\hline$R$ & $\mathrm{~m}$ & 1.006 & 1.135 \\
$B$ & $\mathrm{~T}$ & 0.14 & 0.12 \\
$p$ & $\mathrm{MeV} / \mathrm{c}$ & 41.2 & 41.2 \\
$\sigma_{p}$ & & $1.9 \times 10^{-2}$ & $4.8 \times 10^{-3}$ \\
$\sigma_{x}$ & $\mathrm{~m}$ & $2.4 \times 10^{-3}$ & $4.9 \times 10^{-4}$ \\
$\sigma_{y}$ & $\mathrm{~m}$ & $2.4 \times 10^{-3}$ & $4.9 \times 10^{-4}$ \\
$\sigma_{p_{x}}$ & & $2.7 \times 10^{-4}$ & $1.7 \times 10^{-5}$ \\
$\sigma_{p_{y}}$ & & $2.6 \times 10^{-4}$ & $1.7 \times 10^{-5}$ \\
$\tilde{v}_{x} / c$ & & $2.2 \times 10^{-2}$ & $1.4 \times 10^{-3}$ \\
$\tilde{v}_{y} / c$ & & $2.1 \times 10^{-2}$ & $1.4 \times 10^{-3}$ \\
$\sigma_{s}$ & $\mathrm{fs}$ & 286 & 32 \\
\hline \hline
\end{tabular}

individual particles the interaction between nearest neighbors will dominate [25]. This may contribute to the effect of emerging grainy substructures in a bunch whereby microbunching (see [26], amongst others) is the most prominent of those effects.

Due to the motion of particles a beam can be considered as a thermal distribution. Via $\gamma m \tilde{v}_{x}^{2}=k_{B} T$ we can then assign a transverse temperature $T$ to it. Whether we choose $\tilde{v}_{x}$ or $\tilde{v}_{y}$ as the transverse velocity does not matter if $\left(\tilde{v}_{x}-\tilde{v}_{y}\right) / \tilde{v}_{x} \ll 1$. The latter is the case for the $3 \mathrm{nC}$ and the $1 \mathrm{pC}$ distribution that are considered. We then obtain

$$
\begin{gathered}
T= \begin{cases}2.3 \times 10^{8} \mathrm{~K} & \text { for } 3 \mathrm{nC}, \\
8.8 \times 10^{5} \mathrm{~K} & \text { for } 1 \mathrm{pC},\end{cases} \\
\lambda_{D}= \begin{cases}3.4 \times 10^{-2} \mathrm{~m} & \text { for } 3 \mathrm{nC}, \\
7.9 \times 10^{-3} \mathrm{~m} & \text { for } 1 \mathrm{pC},\end{cases}
\end{gathered}
$$

for the temperature $T$ and the Debye length $\lambda_{D}$. The average interparticle distance $l_{p}$ and the number $N_{p}$ of particles inside a sphere with radius $\lambda_{D}$ is given by

$$
\begin{gathered}
l_{p}= \begin{cases}5.4 \times 10^{-7} \mathrm{~m} \text { for } 3 \mathrm{nC}, \\
1.3 \times 10^{-6} \mathrm{~m} \text { for } 1 \mathrm{pC},\end{cases} \\
N_{p}= \begin{cases}9.8 \times 10^{14} & \text { for } 3 \mathrm{nC}, \\
9.0 \times 10^{11} & \text { for } 1 \mathrm{pC} .\end{cases}
\end{gathered}
$$

We see that the Debye length is 1 order of magnitude larger than the beam radius (compare to $\sigma_{x}$ or $\sigma_{y}$ in Table II) directly before the fourth magnet. Besides, $\lambda_{D} \gg l_{p}$ and $N_{p} \gg 1$. Under these conditions the interaction of a single particle with other particles due to space charge effects can be described by considering a smooth particle distribution. 
Furthermore when the space charge forces become small compared to the external forces, the transverse beam density profile is expected to be Gaussian with respect to the radial distance $r$ from the beam center, cf. Eq. (4.8) in [25]. This can be checked to be the case for our bunches when tracked with Astra. Nevertheless the most reasonable possibility to start with is to describe a particle bunch before the fourth magnet of the FLUTE bunch compressor as a uniformly charged distribution within one sigma in all three spatial dimensions. Besides, for simplicity the charge density is assumed to have sharp edges.

As a measure for the net radial force on particles in a uniform cylindric beam without any external fields the dimensionless generalized perveance $K$ can be introduced. For $K>0$ the beam particles are pushed outwards in the radial direction, which leads to an increase of the beam radius. For $K<0$ the opposite happens and the beam size becomes smaller. The latter can only occur when there are particles inside the beam of opposite charge that neutralize themselves. Especially for FLUTE the generalized perveance is given by

$$
K=\frac{\omega_{p}^{2} r_{m}^{2}}{2 \beta^{2} c^{2}}= \begin{cases}1.2 \times 10^{-6} & \text { for } 3 \mathrm{nC} \\ 3.5 \times 10^{-9} & \text { for } 1 \mathrm{pC} .\end{cases}
$$

We see that for both types of bunches $K \ll 1$ indicating that space charge forces are expected to be weak.

To summarize, all the previous simple estimates demonstrate that space charge forces are of minor influence right before the fourth chicane magnet. However one has to keep in mind that this conclusion results from a rough and simple estimate, where external electric and magnetic fields are neglected. The estimate gives a first idea on the importance of space charge forces within a typical bunch at FLUTE, though. In what follows, the behavior of a particle bunch inside the FLUTE chicane shall be examined in more detail. To do so we make the following assumptions: (1) The charge distribution is continuous. Effects from individual electrons or clusters of electrons are neglected. Therefore changes of the electric and magnetic fields induced by fluctuations of charge density are neglected as well. (2) A cylindrical shape of the bunch is assumed (see Fig. 6), i.e., distortions from this cylindrical shape such as a flat beam are neglected. (3) The changes in bunch dimensions are defined by a single, outermost (boundary) particle. (4) The charge distribution is homogeneous inside the cylinder and falls off to zero directly outside of the cylinder, i.e., it has sharp edges.

In general, particles moving inside the beam pipe are subject to the Lorentz force that originates both from internal and external electromagnetic fields. Internal fields are those that are generated by the charged particles themselves, whereas the external fields are generated by the accelerator, e.g., cavities, dipole magnets, etc. The relativistic equations of motion for an electron moving along a trajectory $\mathbf{r}(t)$ are given by

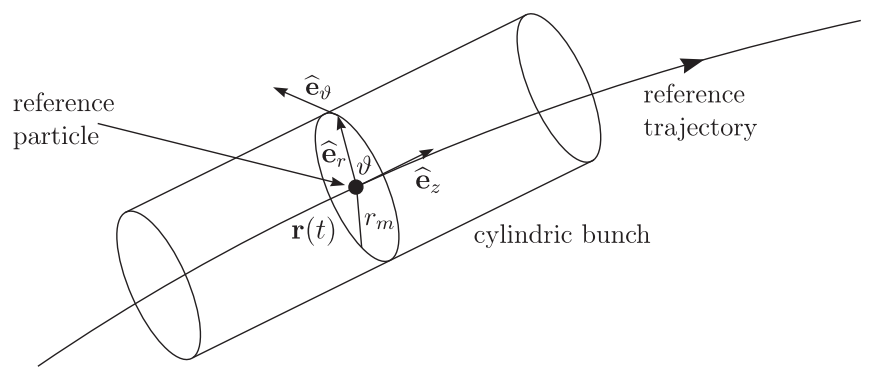

FIG. 6. Bunch traveling along a reference trajectory parametrized by $\mathbf{r}(t)$. We assume the bunch to be of cylindric shape. The coordinates of a bunch particle are described by a cylindric, orthogonal coordinate system whose origin corresponds to the position of the reference particle. The coordinate system is spanned by the basis vectors $\hat{\mathbf{e}}_{r}, \hat{\mathbf{e}}_{\vartheta}$, and $\hat{\mathbf{t}}$. The first points in the radial direction, the second in the circular direction, and the third tangentially to the reference trajectory. The beam radius is called $r_{m}$.

$$
\frac{\mathrm{d}}{\mathrm{d} t}(\gamma(t) m \dot{\mathbf{r}})_{i}=\dot{\gamma} m \dot{\mathbf{r}}_{i}+\gamma m \ddot{\mathbf{r}}_{i}=q(\mathbf{E}+\dot{\mathbf{r}} \times \mathbf{B})_{i},
$$

with the Lorentz factor $\gamma$, the electric field vector $\mathbf{E}$, and the magnetic field vector $\mathbf{B}$. To set up the coordinate system shown in Fig. 6 we need the Frenet trihedron $\{\hat{\mathbf{t}}, \hat{\mathbf{b}}, \hat{\mathbf{n}}\}$ of a general curve. This is made up of the tangent vector $\hat{\mathbf{t}}$, the normal vector $\hat{\mathbf{n}}$, and the binormal vector $\hat{\mathbf{b}}$. These vectors are unit vectors. For their derivatives with respect to time $t$ the Frenet equations hold:

$\dot{\hat{\mathbf{t}}}=|\dot{\mathbf{r}}| \kappa \hat{\mathbf{n}}, \quad \dot{\hat{\mathbf{n}}}=|\dot{\mathbf{r}}|(\tau \hat{\mathbf{b}}-\kappa \hat{\mathbf{t}}), \quad \dot{\hat{\mathbf{b}}}=-|\dot{\mathbf{r}}| \tau \hat{\mathbf{n}}$

where $\kappa=\kappa(t)$ is the curvature and $\tau=\tau(t)$ the torsion of the curve:

$\kappa(t)=\frac{|\dot{\hat{\mathbf{t}}}(t)|}{|\dot{\mathbf{r}}(t)|}=\frac{|\dot{\mathbf{r}}(t) \times \ddot{\mathbf{r}}(t)|}{|\dot{\mathbf{r}}(t)|^{3}}, \quad \tau(t)=\frac{[\dot{\mathbf{r}}(t) \times \ddot{\mathbf{r}}(t)] \cdot \mathbf{r}(t)}{|\dot{\mathbf{r}}(t) \times \ddot{\mathbf{r}}(t)|^{2}}$.

We now consider the propagation of an electron bunch inside a dipole magnet with constant magnetic field pointing in the positive $y$-direction. We split the trajectories of the bunch particles in the reference trajectory $\mathbf{r}_{0}(t)$ plus the coordinates $\mathbf{r}_{b}(t)$ of each particle with respect to the reference particle:

$$
\mathbf{r}(t)=\mathbf{r}_{0}(t)+\mathbf{r}_{b}(t) .
$$

The reference particle is supposed to be situated in the center of the bunch. The equations of motion can then be written in the following form: 
$\frac{\mathrm{d}}{\mathrm{d} t}\left(\gamma m \dot{\mathbf{r}}_{0}\right)+\frac{\mathrm{d}}{\mathrm{d} t}\left(\gamma m \dot{\mathbf{r}}_{b}\right)=q\left[\mathbf{E}+\left(\dot{\mathbf{r}}_{0}+\dot{\mathbf{r}}_{b}\right) \times \mathbf{B}\right]$

Writing the electric and magnetic field as a sum of an internal and an external contribution according to

$$
\begin{aligned}
& \mathbf{E}=\mathbf{E}^{\text {int }}+\mathbf{E}^{\text {ext }}, \\
& \mathbf{B}=\mathbf{B}^{\text {int }}+\mathbf{B}^{\text {ext }},
\end{aligned}
$$

we obtain

$$
\begin{aligned}
& \underbrace{\frac{\mathrm{d}}{\mathrm{d} t}\left(\gamma m \dot{\mathbf{r}}_{0}\right)-q\left(\mathbf{E}^{\mathrm{ext}}+\dot{\mathbf{r}}_{0} \times \mathbf{B}^{\mathrm{ext}}\right)}_{=0}+\frac{\mathrm{d}}{\mathrm{d} t}\left(\gamma m \dot{\mathbf{r}}_{b}\right) \\
& =q\left[\mathbf{E}^{\mathrm{int}}+\dot{\mathbf{r}}_{b} \times\left(\mathbf{B}^{\mathrm{int}}+\mathbf{B}^{\mathrm{ext}}\right)+\dot{\mathbf{r}}_{0} \times \mathbf{B}^{\mathrm{int}}\right] .
\end{aligned}
$$

On the left-hand side of the latter equation the equations of motion of the reference particle can be found, which is assumed to be fulfilled by the trajectory $\mathbf{r}_{0}$. The last term on the right-hand side vanishes, since at the points of the trajectory where the tangent vectors $\dot{\mathbf{r}}_{0}$ are attached to, the internal magnetic field vanishes (see below). In other words, the internal magnetic field vanishes on the trajectory of the reference particle.

We now intend to consider the behavior of the particles that move with a velocity with respect to the reference particle. To derive the equations of motion, the reference trajectory is needed. In a dipole magnet with a constant magnetic field strength vector pointing along the positive $y$ axis it holds that

$$
\mathbf{r}(t)=R\left(\begin{array}{c}
\cos \left(\omega_{0} t\right) \\
0 \\
\sin \left(\omega_{0} t\right)
\end{array}\right), \quad \omega_{0}=\frac{q B}{\gamma m}
$$

where $\omega_{0}$ is the cyclotron frequency and $B$ is the magnetic flux density. For this particular curve the Frenet trihedron is given by

$$
\begin{aligned}
\hat{\mathbf{t}}(t) & =\left(\begin{array}{c}
-\sin \left(\omega_{0} t\right) \\
0 \\
\cos \left(\omega_{0} t\right)
\end{array}\right), \quad \hat{\mathbf{n}}(t)=-\left(\begin{array}{c}
\cos \left(\omega_{0} t\right) \\
0 \\
\sin \left(\omega_{0} t\right)
\end{array}\right), \\
\hat{\mathbf{b}}(t) & =\left(\begin{array}{c}
0 \\
-1 \\
0
\end{array}\right),
\end{aligned}
$$

and we obtain $\kappa(t)=1 / R, \tau(t)=0, \dot{\kappa}(t)=0$, and $\dot{\tau}(t)=0$ for the curvature, torsion, and their derivatives. The modulus of the velocity of a bunch particle with respect to the reference particle is

$$
v_{b} \equiv\left|\dot{\mathbf{r}}_{b}\right|=\sqrt{\dot{r}^{2}+r^{2} \dot{\vartheta}^{2}+\dot{z}^{2}} .
$$

The acceleration $a_{b}$ yields then

$a_{b} \equiv \frac{\mathrm{d}\left|\dot{\mathbf{r}}_{b}\right|}{\mathrm{d} t}=\frac{\mathrm{d} v_{b}}{\mathrm{~d} t}=\frac{1}{v_{b}}\left[\dot{r} \ddot{r}+r \dot{r} \dot{\vartheta}^{2}+r^{2} \dot{\vartheta} \ddot{\vartheta}+\dot{z} \ddot{z}\right]$.

Please note that $v_{b} \neq v$ where $v$ is the velocity of the reference particle, i.e., $v_{b} \ll v$. Using this information the equations of motion for an electron moving inside the magnetic field of a dipole magnet can be obtained where the calculational details are relegated to Appendix B. They read as follows:

$$
\begin{aligned}
& \dot{\gamma} m\left(\dot{r}+\frac{v_{b}}{R} z \cos \vartheta\right)+\gamma m\left[\ddot{r}+\left(\frac{a_{b}}{R} z+\frac{2 v_{b}}{R} \dot{z}\right) \cos \vartheta\right. \\
& \left.-r\left(\dot{\vartheta}^{2}+\frac{v_{b}^{2}}{R^{2}} \cos ^{2} \vartheta\right)\right] \\
& =-e\left[E_{r}^{\mathrm{int}}-v B_{\vartheta}^{\mathrm{int}}+\left(\frac{v_{b}}{R} r \cos \vartheta-\dot{z}\right)\left(B_{\vartheta}^{\mathrm{int}}+B_{\vartheta}^{\mathrm{ext}}\right)\right. \\
& \left.+\left(r \dot{\vartheta}-\frac{v_{b}}{R} z \sin \vartheta\right) B_{t}^{\mathrm{int}}\right] \text {, } \\
& \dot{\gamma} m\left(r \dot{\vartheta}-\frac{v_{b}}{R} z \sin \vartheta\right)+\gamma m\left[r\left(\ddot{\vartheta}+\frac{v_{b}^{2}}{2 R^{2}} \sin (2 \vartheta)\right)+2 \dot{r} \dot{\vartheta}\right. \\
& \left.-\left(\frac{2 v_{b}}{R} \dot{z}+\frac{a_{b}}{R} z\right) \sin \vartheta\right] \\
& =-e\left[E_{\vartheta}^{\mathrm{int}}+v B_{r}^{\mathrm{int}}+\left(\dot{z}-\frac{v_{b}}{R} r \cos \vartheta\right)\left(B_{r}^{\mathrm{int}}+B_{r}^{\mathrm{ext}}\right)\right. \\
& \left.-\left(\dot{r}+\frac{v_{b}}{R} z \cos \vartheta\right) B_{t}^{\mathrm{int}}\right] \text {, } \\
& \dot{\gamma} m\left(\dot{z}-\frac{v_{b}}{R} r \cos \vartheta\right)+\gamma m\left[\ddot{z}-\frac{2 v_{b}}{R} \dot{r} \cos \vartheta-\frac{v_{b}^{2}}{R^{2}} z\right. \\
& \left.+r\left(\frac{2 v_{b}}{R} \dot{\vartheta} \sin \vartheta-\frac{a_{b}}{R} \cos \vartheta\right)\right] \\
& =-e\left[E_{t}^{\mathrm{int}}+\left(\dot{r}+\frac{v_{b}}{R} z \cos \vartheta\right)\left(B_{\vartheta}^{\mathrm{int}}+B_{\vartheta}^{\mathrm{ext}}\right)\right. \\
& \left.-\left(r \dot{\vartheta}-\frac{v_{b}}{R} z \sin \vartheta\right)\left(B_{r}^{\mathrm{int}}+B_{r}^{\mathrm{ext}}\right)\right] \text {. }
\end{aligned}
$$

No approximations have been made so far, i.e., the latter three equations are exact. Since there is no external electric field accelerating the particles we use $\dot{\gamma}=0$ and $\dot{v}=0$.

According to [25] we introduce dimensionless functions as follows:

$$
r(t)=r_{0} \varrho(\xi), \quad \vartheta(t)=\varphi(\xi), \quad z(t)=L \zeta(\xi),
$$




$$
l=l_{0} \xi, \quad l_{0}=\frac{r_{0}}{\sqrt{2 K}}, \quad K=\frac{e I}{2 \pi \varepsilon_{0} m(c \beta \gamma)^{3}} .
$$

Here $r_{0}$ is the initial radial particle distance to the cylinder axis and $L$ the initial cylinder length, ${ }^{2}$ which both are characteristic length scales of the problem considered. We express the traveled distance $l$ of the bunch via $r_{0}$ as well. ${ }^{3}$ $K$ is the dimensionless generalized perveance. Taking $\dot{l}=v$ into account with the velocity $v$ of the reference particle, the derivatives of the functions can be expressed via dimensionless derivatives and the length scales previously introduced. Furthermore, we use the notation $\tilde{\mathbf{E}}(\xi) \equiv \mathbf{E}[t(\xi)]$, $\tilde{\mathbf{B}}(\xi) \equiv \mathbf{B}[t(\xi)], \tilde{v}(\xi) \equiv v[t(\xi)]$, and $\tilde{\gamma}(\xi)=\gamma[t(\xi)]$ for the corresponding functions in terms of the dimensionless variable $\xi$. We then obtain

$$
\begin{gathered}
\dot{r}=\frac{\mathrm{d} r}{\mathrm{~d} t}=\dot{l} \frac{\mathrm{d} r}{\mathrm{~d} l}=\frac{\dot{l} r_{0}}{l_{0}} \frac{\mathrm{d} \varrho}{\mathrm{d} \xi}=v \sqrt{2 K} \varrho^{\prime}(\xi), \\
\ddot{r}=\dot{v} \sqrt{2 K} \varrho^{\prime}(\xi)+2 K \frac{v^{2}}{r_{0}} \varrho^{\prime \prime}(\xi), \\
\dot{\vartheta}=\frac{v}{l_{0}} \varphi^{\prime}(\xi)=\frac{v}{r_{0}} \sqrt{2 K} \varphi^{\prime}(\xi), \\
\ddot{\vartheta}=\frac{\dot{v}}{r_{0}} \sqrt{2 K} \varphi^{\prime}(\xi)+2 K\left(\frac{v}{r_{0}}\right)^{2} \varphi^{\prime \prime}(\xi), \\
\dot{z}=\frac{v L}{l_{0}} \zeta^{\prime}(\xi)=v \sqrt{2 K} \frac{L}{r_{0}} \zeta^{\prime}(\xi), \\
\ddot{z}(t)=\frac{\dot{v} L}{r_{0}} \sqrt{2 K} \zeta^{\prime}(\xi)+2 K\left(\frac{v}{r_{0}}\right)^{2} L \zeta^{\prime \prime}(\xi), \\
\dot{v}=\dot{l} \frac{\mathrm{d} v}{\mathrm{~d} l}=\sqrt{2 K} \frac{\tilde{v}}{r_{0}} \tilde{v}^{\prime}(\xi), \\
\dot{\gamma}=\dot{l} \frac{\mathrm{d} \tilde{\gamma}}{\mathrm{d} l}=\sqrt{2 K} \frac{\tilde{v}}{r_{0}} \tilde{\gamma}^{\prime}(\xi) .
\end{gathered}
$$

The dimensionless equations of motion containing the general internal and external electric and magnetic fields can be found in Eqs. (B5)-(B7).

We now employ the following assumptions for a first simplification of the equations of motion. A cylindric bunch with length $L$, homogeneous charge $Q=-Q_{b}$ with $Q_{b}>0$, and velocity $v \geq 0$ can be associated with the current $I=-I_{b}=-Q_{b} v / L$ (with $I_{b}>0$ ). Such a bunch current produces an electric field pointing in radial direction and a magnetic field pointing in circular direction. They are given by (see, e.g., [25])

\footnotetext{
${ }^{2}$ With $L$ we mean the full length of the cylinder.

${ }^{3}$ This choice is in accordance with [25]; in principle $L$ could also be used.
}
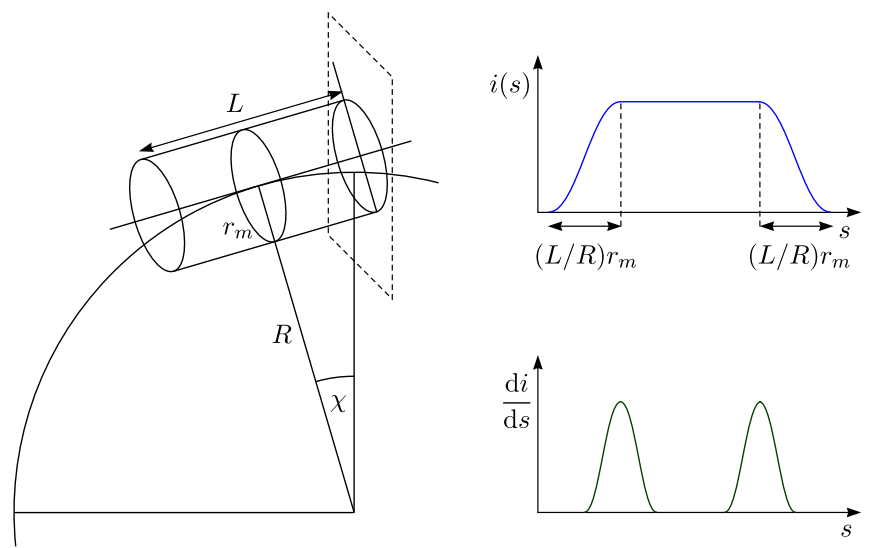

FIG. 7. Curvature effects for internal electromagnetic fields in a cylindrical bunch. The curves on the right-hand side show the current density and its first derivative as functions of the longitudinal coordinate. Upon propagation through a fixed plane the current density changes when the cylinder frames enter and exit the plane, respectively. These changes happen within distances of the order of magnitude of $(L / R) r_{m}$ with the bunch radius $r_{m}$.

$$
\begin{aligned}
& \mathbf{E}^{\text {int }}(r)=-\frac{I_{b}}{2 \pi \varepsilon_{0} v} \frac{r}{r_{m}^{2}} \hat{\mathbf{e}}_{r}, \\
& \mathbf{B}^{\text {int }}(r)=-\frac{\mu_{0} I_{b}}{2 \pi} \frac{r}{r_{m}^{2}} \hat{\mathbf{e}}_{\vartheta}, \quad r \leq r_{m},
\end{aligned}
$$

where $\varepsilon_{0}$ is the vacuum permittivity, $\mu_{0}$ the vacuum permeability, and $r_{m}$ the radius of the cylinder. The distance from the symmetry axis of the bunch is given by $r$. The unit vector pointing in the radial direction is $\hat{\mathbf{e}}_{r}$ and the unit vector in the circular direction is $\hat{\mathbf{e}}_{\vartheta}$. From Eq. (B4) we see that the internal fields are mainly involved in the $r$ component of the Lorentz force.

The form of the internal electric and magnetic fields of Eq. (4.20) is valid on straight trajectories. Concerning the kinematics on curved orbits, the situation is as follows. A possible change of the internal fields in comparison to a straight orbit arises due to the cylinder fringes, cf. Fig. 7. When a bunch traverses a plane perpendicular to the orbit, the current density is then not homogeneous any more. The reason is that the cylinder front enters the plane under a nonvanishing angle $\chi$. Hence the current density grows continuously to a constant value when the bunch enters the plane and it decreases continuously to zero again upon exiting the plane. To estimate the size of this effect, consider a circle with radius $R$ and constant curvature $\kappa=1 / R$. When the bunch touches the plane first, the angle $\chi$ lies in the order of magnitude of $L /(2 R)$. We will see that for a typical bunch considered this value is much smaller than 1 , which is why such effects can be safely neglected (otherwise than within the approach considered in [27]).

Longitudinal curvature effects on the energy change of a bunch caused by space charge forces are described, e.g., in 
Eq. (12) of [28]; note the term proportional to $1 / \gamma^{2}$ resulting from Coulomb forces. The integrand involves the derivative of the longitudinal bunch form factor $\lambda=$ $\lambda(s)$ with respect to time, which is nonzero for a nontrivial form factor. However the longitudinal form factor that we use is a mere superposition of Heaviside step functions. Therefore the derivative will deliver nonzero contributions from the cylinder fringes only, which are precisely the suppressed effects covered in the previous paragraph.

With $\dot{\mathbf{r}}_{0}=v \hat{\mathbf{t}}$ Eq. (4.20) leads to the following Lorentz force acting on an electron with charge $q=-e$ :

$$
\begin{aligned}
-e\left(\mathbf{E}^{\mathrm{int}}-\dot{\mathbf{r}}_{0} \times \mathbf{B}^{\mathrm{int}}\right)_{r} & =-e\left(E_{r}^{\mathrm{int}}-v B_{\vartheta}^{\mathrm{int}}\right) \\
& =e\left(\frac{I_{b}}{2 \pi \varepsilon_{0} v} \frac{r}{r_{m}^{2}}-v \cdot \frac{\mu_{0} I_{b}}{2 \pi} \frac{r}{r_{m}^{2}}\right) \\
& =\frac{e I_{b}}{2 \pi \varepsilon_{0} v} \frac{r}{r_{m}^{2}}\left(1-\frac{v^{2}}{c^{2}}\right) \\
& =\frac{e I_{b}}{2 \pi \varepsilon_{0} v} \frac{r}{r_{m}^{2}} \frac{1}{\gamma^{2}} .
\end{aligned}
$$

Hence, the space charge forces that a particle in a homogeneous cylindric bunch feels along the radial direction are suppressed by a factor $1 / \gamma^{2}$. As a next step we assume that the remaining internal field components are negligible, i.e.,

$$
\tilde{E}_{\vartheta}^{\mathrm{int}}=\tilde{E}_{t}^{\mathrm{int}}=\tilde{B}_{r}^{\mathrm{int}}=\tilde{B}_{t}^{\mathrm{int}}=0 .
$$

The velocity and acceleration of bunch particles in dimensionless coordinates result from Eqs. (4.15) and (4.16) and read as follows:

$$
\begin{aligned}
& \tilde{v}_{b}=\sqrt{2 K} \tilde{v} f[\varrho, \varphi, \zeta], \\
& \tilde{a}_{b}=\frac{2 K \tilde{v} \tilde{v}^{\prime}}{r_{0}} f[\varrho, \varphi, \zeta]+\frac{2 K \tilde{v}^{2}}{r_{0}} g[\varrho, \varphi, \zeta], \\
& f[\varrho, \varphi, \zeta] \equiv \sqrt{\varrho^{\prime 2}+\varrho^{2} \varphi^{\prime 2}+\left(L / r_{0}\right)^{2} \zeta^{\prime 2}},
\end{aligned}
$$

$g[\varrho, \varphi, \zeta] \equiv \frac{\varrho^{\prime} \varrho^{\prime \prime}+\varrho Q^{\prime} \varphi^{2}+\varrho^{2} \varphi^{\prime} \varphi^{\prime \prime}+\left(L / r_{0}\right)^{2} \zeta^{\prime} \zeta^{\prime \prime}}{\sqrt{\varrho^{\prime 2}+\varrho^{2} \varphi^{\prime 2}+\left(L / r_{0}\right)^{2} \zeta^{\prime 2}}}$.

The notation $f=f[\bullet], g=g[\bullet]$ shall indicate that $f, g$ contain the functions given as arguments plus additional derivatives of these functions.

We now express the equations of motion by solely using dimensionless functions. All physical parameters then do not appear in the functions or their derivatives any more but in quantities that are denoted as Greek letters. Furthermore these are numbered according to their order in the differential equations. The differential equation describing the motion of bunch particles in the radial direction of the cylinder in Fig. 6 is given by

$$
\begin{aligned}
& \eta_{1}\left(\varrho^{\prime}+\eta_{2} f \zeta \cos \varphi\right)+\varrho^{\prime \prime}+\eta_{3} \varrho^{\prime} \\
& \quad+\left[\eta_{4}\left(\eta_{3} f+g\right) \zeta+\eta_{5} f \zeta^{\prime}\right] \cos \varphi-\varrho\left(\varphi^{\prime 2}+\eta_{6} f^{2} \cos ^{2} \varphi\right) \\
& =\eta_{7}\left[\eta_{8} \varrho+\left(\eta_{9} f \varrho \cos \varphi-\eta_{10} \zeta^{\prime}\right)\left(\eta_{11} \varrho-\tilde{B}_{\varphi}^{\mathrm{ext}}\right)\right] \\
& \eta_{1}=\frac{\tilde{\gamma}^{\prime}}{\tilde{\gamma}}, \quad \eta_{2}=\frac{L}{R}, \quad \eta_{3}=\frac{\tilde{v}^{\prime}}{\tilde{v}}, \\
& \eta_{4}=\eta_{2}, \quad \eta_{5}=2 \eta_{2}, \\
& \eta_{6}=\frac{r_{0}^{2}}{R^{2}}, \quad \eta_{7}=\frac{e r_{0}^{2}}{2 K \tilde{v}^{2} \tilde{\gamma} m}, \quad \eta_{8}=\frac{I_{b}}{2 \pi \varepsilon_{0} \tilde{v} \tilde{\gamma}^{2} r_{m}^{2}} \\
& \eta_{9}=\frac{\sqrt{2 K} \tilde{v}}{R}, \quad \eta_{10}=\frac{\sqrt{2 K} \tilde{v} L}{r_{0}^{2}}, \quad \eta_{11}=\frac{\mu_{0} I_{b} r_{0}}{2 \pi r_{m}^{2}}
\end{aligned}
$$

Note that both $\eta_{1}$ and $\eta_{3}$ are exactly equal to zero when the norm of the particle velocity is constant. Quantities containing only bunch dimensions or velocities are merely related to kinematics, whereas quantities containing the elementary charge $e$ have to do with space charge forces. Furthermore, the occurrence of the bunch current $I_{b}$ indicates internal electric and magnetic fields that are generated by the bunch itself. The parameter $\eta_{8}$ shows the cancellation of the internal radial electric field and the internal circular magnetic field leaving a remainder proportional to $1 / \gamma^{2}$. This was already indicated in Eq. (4.21).

The differential equation describing the circular motion of bunch particles is as follows:

$$
\begin{gathered}
\chi_{1}\left(\varrho \varphi^{\prime}-\chi_{2} f \zeta \sin \varphi\right)+\varrho\left[\varphi^{\prime \prime}+\chi_{3} \varphi^{\prime}+\chi_{4} f^{2} \sin (2 \varphi)\right] \\
+2 \varrho^{\prime} \varphi^{\prime}-\left[\chi_{5}\left(\chi_{3} f+g\right) \zeta+\chi_{6} f \zeta^{\prime}\right] \sin \varphi \\
=\chi_{7}\left(\chi_{9} f \varrho \cos \varphi-\chi_{10} \zeta^{\prime}\right) \tilde{B}_{Q}^{\mathrm{ext}} \\
\chi_{1}=\frac{\tilde{\gamma}^{\prime}}{\tilde{\gamma}}, \quad \chi_{2}=\frac{L}{R}, \quad \chi_{3}=\frac{\tilde{v}^{\prime}}{\tilde{v}} \\
\chi_{4}=\frac{r_{0}^{2}}{2 R^{2}}, \quad \chi_{5}=\chi_{2} \\
\chi_{6}=2 \chi_{2}, \quad \chi_{7}=\frac{e r_{0}^{2}}{2 K \tilde{v}^{2} \tilde{\gamma} m} \\
\chi_{9}=\frac{\sqrt{2 K} \tilde{v}}{R}, \quad \chi_{10}=\frac{\sqrt{2 K} \tilde{v} L}{r_{0}^{2}}
\end{gathered}
$$

Contrary to Eq. (4.24) this equation of motion involves the radial external magnetic field component instead of the circular one. Furthermore, the internal electric and magnetic fields do not play a role for the circular motion of the particle.

Finally, the differential equation for the motion of the bunch particles in the axial direction of the cylindric bunch reads 


$$
\begin{aligned}
& \psi_{1}\left(\zeta^{\prime}-\psi_{2} f \varrho \cos \varphi\right)+\zeta^{\prime \prime}+\psi_{3} \zeta^{\prime}-\psi_{4} f^{2} \zeta-\psi_{5} f \varrho^{\prime} \cos \varphi \\
& \quad+\varrho\left[\psi_{5} f \varphi^{\prime} \sin \varphi-\psi_{6}\left(\psi_{3} f+g\right) \cos \varphi\right] \\
& =\psi_{7}\left[\left(\psi_{9} \rho^{\prime}+\psi_{10} f \zeta \cos \varphi\right)\left(\psi_{11} \rho-\tilde{B}_{\varphi}^{\mathrm{ext}}\right)\right. \\
& \left.\quad+\left(\psi_{9} \rho \varphi^{\prime}-\psi_{10} f \zeta \sin \varphi\right) \tilde{B}_{Q}^{\mathrm{ext}}\right], \\
& \psi_{1}=\frac{\tilde{\gamma}^{\prime}}{\tilde{\gamma}}, \quad \psi_{2}=\frac{r_{0}^{2}}{R L}, \quad \psi_{3}=\frac{\tilde{v}^{\prime}}{\tilde{v}}, \\
& \psi_{4}=\frac{r_{0}^{2}}{R^{2}}, \quad \psi_{5}=2 \psi_{2}, \\
& \psi_{6}=\psi_{2}, \quad \psi_{7}=\frac{e r_{0}^{2}}{2 K \tilde{v}^{2} \tilde{\gamma} m}, \quad \psi_{9}=\frac{\sqrt{2 K} \tilde{v}}{L} \\
& \psi_{10}=\frac{\sqrt{2 K} \tilde{v}}{R}, \quad \psi_{11}=\frac{\mu_{0} I_{b} r_{0}}{2 \pi r_{m}^{2}} .
\end{aligned}
$$

The quantities $\left\{\eta_{1}, \ldots, \eta_{6}, \eta_{7} \times\left\{\eta_{8}, B \eta_{9}, B \eta_{10}, \eta_{9} \eta_{11}, \eta_{10} \eta_{11}\right\}\right\}$, $\left\{\chi_{1}, \ldots, \chi_{6}, B \chi_{7} \times\left\{\chi_{9}, \chi_{10}\right\}\right\}, \quad$ and $\quad\left\{\psi_{1}, \ldots, \psi_{6}, \psi_{7} \times\right.$ $\left.\left\{B \psi_{9}, B \psi_{10}, \psi_{9} \psi_{11}, \psi_{10} \psi_{11}\right\}\right\}$ with the modulus of the external magnetic flux density $B$ are dimensionless. The numbering of the coefficients has been performed such that a correspondence between coefficients of different equations of motion is evident. The first six coefficients of each differential equation are related to the kinematics; they only involve kinematic quantities such as beam dimensions and velocities. The product of the seventh and eighth coefficient in Eq. (4.24) describes the space charge effects due to the internal electric and magnetic field. The fact that no $\chi_{8}$ appears in Eq. (4.25) and no $\psi_{8}$ in Eq. (4.26) demonstrates that this special kind of force does not appear in the circular and the longitudinal equation of motion.

\section{A. Space charge effects in the FLUTE bunch compressor}

In the calculations of the previous section none of the terms in the equations of motion were neglected a priori. We will now estimate the order of magnitude of the related quantities for the FLUTE chicane such that they can be compared with each other. First of all, certain physical values, e.g., the beam size or the beam current depend on the bunch charge considered. We decided to compare the two extreme beam dynamics cases that were simulated with Astra: a bunch with the high charge of $3 \mathrm{nC}$ and a bunch with the very low charge of $1 \mathrm{pC}$.

Furthermore one has to keep in mind that the bunch properties are not constant in the chicane. For example during the process of bunch compression the peak current will increase. That is why as a simple estimate of the behavior of the bunch due to space charge forces we take the initial values right before the fourth chicane magnet. Another important point is that each bunch is a smeared-out particle distribution. Hence, it has no sharp edges opposite to the pictorial representation of the cylindric bunch in

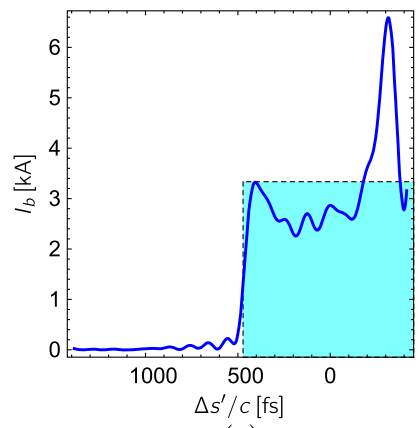

(a)

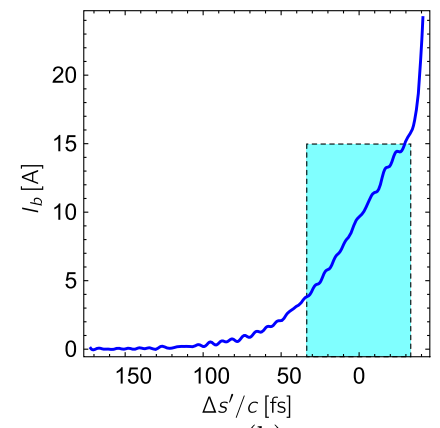

(b)
FIG. 8. Bunch currents $I_{b}$ of the $3 \mathrm{nC}$ (a) and $1 \mathrm{pC}$ bunch (b) right before the fourth chicane magnet as a function of the longitudinal electron distance $\Delta s^{\prime} / c$ with respect to the bunch center. Here the coordinates have been corrected by a factor of $1 / \cos (\alpha)$ where $\alpha$ is the bending angle of the dipole magnet. The points are connected by interpolation to remove small-scale oscillations caused by the binning. The two major peaks in the longitudinal current distribution in (a) originate mainly from the space charge influenced extraction from the cathode and subsequent velocity deviations induced by rf fields in the gun. The net result of those nonlinear mechanisms in the corresponding $3 \mathrm{nC}$ bunch can already be seen in Fig. 3(a) and becomes more pronounced after compression, see Fig. 3(c). The cylinders that will be taken as replacement bunches in the analytical approach are marked by dashed lines [see the paragraph below Eq. (4.27) for how to obtain these cylinders]. For (a) a binning of $50 \mathrm{fs}$ is chosen resulting in $I_{\text {peak }}=3260 \mathrm{~A}$ and for (b) a binning of $3 \mathrm{fs}$ is used whereby $I_{\text {peak }}=15.4 \mathrm{~A}$.

Fig. 6. For this reason we take the corresponding rms values, e.g., the rms beam size ${ }^{4}$ for the radius $r_{m}$ and $\Upsilon$ times the rms bunch length $\sigma_{s}^{(4 \text { th) }}$ (before the fourth magnet) for the cylinder length $L$.

$$
r_{m} \equiv \sqrt{\sigma_{x} \sigma_{y}}, \quad L \equiv \Upsilon \sigma_{s}^{(4 \mathrm{th})}
$$

In this section the cylinder length is not simply taken as the double of the rms bunch length, which was done within the simple estimates at the beginning of the chapter. Now the bunch currents will be plotted as functions of the longitudinal electron distance with respect to the bunch center (divided by $c$ ). It is reasonable to obtain the parameter $\Upsilon$ from these plots based on the procedure stated in the following lines. As the bunch current for $3 \mathrm{nC}$ strongly decreases for $\Delta s^{\prime} / c \lesssim-430$ fs [see Fig. 8(a)], the cylindrical equivalent is chosen such that its length ranges over all $\Delta s^{\prime} / c$ where the bunch current is larger than around $1 \mathrm{kA}$. Therefore we choose $\Upsilon \approx 3.0$ for $3 \mathrm{nC}$. This value is supposed to describe the cylindrical equivalent of the $3 \mathrm{nC}$ bunch much better than $\Upsilon=2$ that we chose for simplicity

\footnotetext{
${ }^{4}$ According to the charge density $n$ given in Eq. (4.1) it makes sense to obtain the beam size as the geometric average of the transverse beam sizes $\sigma_{x}$ and $\sigma_{y}$.
} 
at the beginning of the chapter. Apart from one major fluctuation the current profile is quite uniform between the center and the front of the bunch and it drops to zero quickly for $\Delta s^{\prime} / c \lesssim-430 \mathrm{fs}$. The front is characterized by a high peak showing that a large number of electrons gathers there. Since the width of this peak is rather narrow compared to the overall bunch length and it cannot be dealt with in our simple analytical model, it will not be taken into account. The current profile of the $1 \mathrm{pC}$ bunch in Fig. 8(b) does not have a regime where a constant current could be called a reasonable approximation. Instead it increases steadily to its maximum value over the whole range of longitudinal positions. Since a better option does not seem to be at hand, we will stick to $\Upsilon=2$ for $1 \mathrm{pC}$.

The bending radius is chosen from the design values in [6]. The current directly follows from the tracked bunch data using an appropriate binning (see Fig. 8). Such a bunch consists of $N_{p}=5 \times 10^{4}$ macroparticles. Counting the number of macroparticles inside a bin, multiplying it with $Q_{b} / N_{p}$ (where $Q_{b}$ is the bunch charge) and dividing the product by the bin size leads to the current in terms of the longitudinal coordinate of the bunch. We then define the peak current $I_{\text {peak }}$ of a bunch as

$$
I_{\text {peak }} \equiv \frac{Q_{b}}{L}=\frac{Q_{b}}{\Upsilon \sigma_{s}^{(4 \mathrm{th})}} .
$$

The Alfvén current $I_{A}$ is the maximum current possible for a collimated, cylindrical beam of charged particles under the influence of space charge effects. It can be written with the characteristic current $I_{0}$ as follows [29]:

$$
I_{A}=I_{0} \beta \gamma, \quad I_{0}=\frac{4 \pi \varepsilon_{0} m c^{3}}{e} .
$$

The characteristic current is the part of the Alfvén current that is not related to the kinematics of the beam. The peak current of the bunch normalized by $I_{0}$ approximately corresponds to the Budker parameter $\nu_{B}$ for relativistic particles $[25,29]$. According to the peak current obtained in Fig. 8 the Budker parameter is given by

$$
\nu_{B} \equiv \frac{I_{\text {peak }}}{I_{0} \beta} \approx \frac{I_{\text {peak }}}{I_{0}}= \begin{cases}1.9 \times 10^{-1} & \text { for } 3 \mathrm{nC}, \\ 9.1 \times 10^{-4} & \text { for } 1 \mathrm{pC} .\end{cases}
$$

We see that for the $1 \mathrm{pC}$ bunch at FLUTE the peak current is much smaller than the characteristic current and even more than the Alvén current (because of the Lorentz factor). So we are far away from the regime where the beam may become unstable due to space charge forces. This is what happens only for currents that lie in the vicinity of $I_{A}$. However, for the $3 \mathrm{nC}$ bunch the peak current is, indeed, smaller than $I_{0}$ but not negligibly small. This may have some influence on the treatment of space charge effects and we will come back to this issue at the end of the current chapter. Note that also a geometrical factor due to the beam shape may shift the effective Budker parameter, which will not be considered further, though. Using the definition of $\nu_{B}$ in Eq. (4.30), the generalized perveance $K$ can also be computed as follows:

$$
K=\frac{I_{\text {peak }}}{I_{0}} \frac{2}{\beta^{3} \gamma^{3}}=\frac{2 \nu_{B}}{\beta^{2} \gamma^{3}},
$$

giving a value for $1 \mathrm{pC}$ that is in accordance with Eq. (4.6). For $3 \mathrm{nC}$ the value of $K$ has to be adapted due to the recent choice $\Upsilon \approx 3.0$.

In Table III all dimensionless quantities appearing in the equations of motion are obtained for both the particular $3 \mathrm{nC}$ and the $1 \mathrm{pC}$ bunch considered. Quantities purely related to curvature effects due to the trajectory are much smaller than 1 , since they involve either ratios $r_{0} / R$ or $L / R$ where the curvature radius $R$ is much larger than the bunch dimensions $r_{0}$ and $L$. This is a proof of principle that the approximation of a cylinder with its center traveling along the trajectory and its axis being tangential to the curve is warranted. Therefore it is also possible to work with Eq. (4.20) to model the internal electric and magnetic fields of the bunch. Bear in mind that the terms in the equations of motion (4.24)-(4.26) that do not appear together with a dimensionless physical quantity such as $\eta_{2}$ are multiplied with 1 . In this context the term including the prefactor $\eta_{7} \eta_{8}=1 / 2$ is characteristic, too. We now

TABLE III. Dimensionless physical parameters as they appear in the equations of motion (4.24)-(4.26). Each pair of columns gives the parameters needed plus their values for FLUTE using Table II and Fig. 8.

\begin{tabular}{lccccccccc}
\hline \hline$Q_{b}$ & $\eta_{2}$ & $\eta_{4}$ & $\eta_{5}$ & $\eta_{6}$ & $\eta_{7} \tilde{\eta}_{8}$ & $\eta_{7} \eta_{9} B$ & $\eta_{7} \eta_{9} \tilde{\eta}_{11}$ & $\eta_{7} \eta_{10} B$ & $\eta_{7} \eta_{10} \tilde{\eta}_{11}$ \\
\hline $3 \mathrm{nC}$ & $2.56 \times 10^{-4}$ & $2.56 \times 10^{-4}$ & $5.13 \times 10^{-4}$ & $5.75 \times 10^{-6}$ & 0.5 & $4.61 \times 10^{-3}$ & $9.74 \times 10^{-3}$ & 0.205 & 0.434 \\
$1 \mathrm{pC}$ & $1.69 \times 10^{-5}$ & $1.69 \times 10^{-5}$ & $3.37 \times 10^{-5}$ & $1.86 \times 10^{-7}$ & 0.5 & $2.21 \times 10^{-3}$ & $1.17 \times 10^{-4}$ & 0.201 & $1.06 \times 10^{-2}$ \\
$Q_{b}$ & $\chi_{2}$ & $\chi_{4}$ & $\chi_{5}$ & $\chi_{6}$ & $\chi_{7} \chi_{9} B$ & $\chi_{7} \chi_{10} B$ & & & \\
$3 \mathrm{nC}$ & $2.56 \times 10^{-4}$ & $2.88 \times 10^{-6}$ & $2.56 \times 10^{-4}$ & $5.13 \times 10^{-4}$ & $4.61 \times 10^{-3}$ & 0.205 & & & \\
$1 \mathrm{pC}$ & $1.69 \times 10^{-5}$ & $9.29 \times 10^{-8}$ & $1.69 \times 10^{-5}$ & $3.37 \times 10^{-5}$ & $2.21 \times 10^{-3}$ & 0.201 & & & \\
$Q_{b}$ & $\psi_{2}$ & $\psi_{4}$ & $\psi_{5}$ & $\psi_{6}$ & $\psi_{7} \psi_{9} B$ & $\psi_{7} \psi_{9} \tilde{\psi}_{11}$ & $\psi_{7} \psi_{10} B$ & $\psi_{7} \psi_{10} \tilde{\psi}_{11}$ & \\
$3 \mathrm{nC}$ & $2.24 \times 10^{-2}$ & $5.75 \times 10^{-6}$ & $4.49 \times 10^{-2}$ & $2.24 \times 10^{-2}$ & 18.0 & 38.0 & $4.61 \times 10^{-3}$ & $9.74 \times 10^{-3}$ & \\
$1 \mathrm{pC}$ & $1.10 \times 10^{-2}$ & $1.86 \times 10^{-7}$ & $2.20 \times 10^{-2}$ & $1.10 \times 10^{-2}$ & 131 & 6.96 & $2.21 \times 10^{-3}$ & $1.17 \times 10^{-4}$ & \\
\hline \hline
\end{tabular}


simplify the equations of motion such that all terms multiplied by a number much smaller than 1 according to Table III are neglected. This leads to a set of simplified differential equations given as follows:

$$
\begin{gathered}
\varrho^{\prime \prime}=\eta_{7}\left[\eta_{8} \varrho-\eta_{10} \zeta^{\prime}\left(\eta_{11} \varrho-\tilde{B}_{\varphi}^{\mathrm{ext}}\right)\right], \\
\varrho \varphi^{\prime \prime}+2 \varrho^{\prime} \varphi^{\prime}=-\chi_{7} \chi_{10} \zeta^{\zeta^{\prime}} \tilde{B}_{\varrho}^{\mathrm{ext}}, \\
\zeta^{\prime \prime}=\psi_{7} \psi_{9}\left[\varrho^{\prime}\left(\psi_{11} \varrho-\tilde{B}_{\varphi}^{\mathrm{ext}}\right)+\varrho \varphi^{\prime} \tilde{B}_{\varrho}^{\mathrm{ext}}\right] .
\end{gathered}
$$

Setting $\tilde{B}_{Q}^{\text {ext }}=\tilde{B}_{\varphi}^{\text {ext }}=0$, the resulting set of equations holds for the drift spaces of the FLUTE chicane. In this case the first of these simplified equations of motion partially decouples from the other two, i.e., the angular variable $\varphi$ does not appear any more. This shows that for mere drifts the circular motion of particles inside the bunch due to the magnetic fields can be neglected when considering the increase of the transverse beam dimensions.

The (constant) external magnetic flux density in the dipole magnet along the positive $y$-direction can be decomposed in a radial and a circular component:

$$
\begin{aligned}
\tilde{\mathbf{B}}^{\mathrm{ext}} & =B\left[\left(\hat{\mathbf{e}}_{y} \cdot \hat{\mathbf{e}}_{\varrho}\right) \hat{\mathbf{e}}_{\varrho}+\left(\hat{\mathbf{e}}_{y} \cdot \hat{\mathbf{e}}_{\varphi}\right) \hat{\mathbf{e}}_{\varphi}+\left(\hat{\mathbf{e}}_{y} \cdot \hat{\mathbf{t}}\right) \hat{\mathbf{t}}\right] \\
& =-B\left[\hat{\mathbf{e}}_{\varrho} \sin \varphi+\hat{\mathbf{e}}_{\varphi} \cos \varphi\right] .
\end{aligned}
$$

The next important issue to mention is that the internal electric and magnetic field components themselves given by Eq. (4.20) depend on the cylinder radius $r_{m}$. Since we are interested in the evolution of $r_{m}$ as a function of time we cannot take it as a constant. Under the assumption that the particle trajectories are laminar, i.e., they do not intersect each other, it suffices to consider the envelope particles. Because of this we set $r_{m}=r_{0} \varrho(\xi)$ with $r_{0}=$ $\sqrt{\sigma_{x} \sigma_{y}}$ being the initial radial distance of an envelope particle to the cylinder axis. This procedure is followed in [25] as well and leads to the final system of differential equations:

$$
\begin{gathered}
\varrho^{\prime \prime}=\eta_{7}\left[\frac{\tilde{\eta}_{8}}{\varrho}-\eta_{10} \zeta^{\prime}\left(\frac{\tilde{\eta}_{11}}{\varrho}+B \cos \varphi\right)\right], \\
\varrho \varphi^{\prime \prime}+2 \varrho^{\prime} \varphi^{\prime}=\chi_{7} \chi_{10} \zeta^{\prime} B \sin \varphi, \\
\zeta^{\prime \prime}=\psi_{7} \psi_{9}\left[\varrho^{\prime}\left(\frac{\tilde{\psi}_{11}}{\varrho}+B \cos \varphi\right)-\varrho \varphi^{\prime} B \sin \varphi\right],
\end{gathered}
$$

with the following definitions where the cylinder radius $r_{m}$ corresponds to the initial radial particle distance $r_{0}$ :

$$
\begin{aligned}
\tilde{\eta}_{8} & =\left.\eta_{8}\right|_{r_{m}=r_{0}}, \quad \tilde{\eta}_{11}=\left.\eta_{11}\right|_{r_{m}=r_{0}}, \\
\tilde{\psi}_{11} & =\left.\psi_{11}\right|_{r_{m}=r_{0}} .
\end{aligned}
$$

If in Eq. (4.34a) we set the external magnetic field $B$ equal to zero and neglect particle motions along the $z$-direction of the coordinate system (resulting in $\zeta^{\prime}=0$ ) we obtain

$$
\varrho^{\prime \prime}=\frac{\eta_{7} \tilde{\eta}_{8}}{\varrho}
$$

This differential equation is discussed at the beginning of the fourth chapter in [25]. The numerical solutions for different initial conditions are presented in Fig. 9. They correspond to the plots given in the latter reference, which is a good cross-check for the method used here. In the figure we see that space charge effects always blow up the radial beam dimension. If the beam is focused, e.g., by magnetic quadrupoles the beam size first decreases until a certain minimum value and then it starts increasing again.

The model considered here is more general in the sense that it does not neglect certain effects at the start of the calculations. The differential equations given by (4.34a)-(4.34c) describe the motion of particles in radial, angular, and $z$-direction with respect to the reference particle. Furthermore external magnetic fields can be taken into account. The equations for a drift space follow by setting $B=0$. Using the values of Table III, the system of differential equations can be solved numerically. This is done for both the $3 \mathrm{nC}$ and the $1 \mathrm{pC}$ bunch right before the fourth magnet of the FLUTE bunch compressor.

We intend to solve the system of differential equations for the following initial conditions:

$$
\begin{array}{cc}
r(t=0)=r_{0}, & \dot{r}(t=0)=0, \\
\vartheta(t=0)=\vartheta_{0}, & \dot{\vartheta}(t=0)=0, \\
z(t=0)=L, & \dot{z}(t=0)=\Delta v .
\end{array}
$$

The first two conditions mean that the initial beam size is $r_{0}$ and the change of the beam size vanishes, which makes

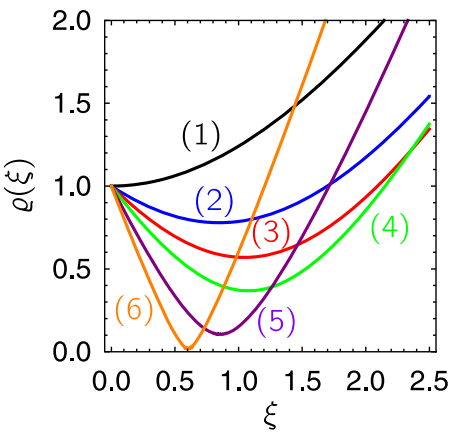

FIG. 9. Behavior of the dimensionless function $\varrho(\xi)$ defined by Eq. (4.18) (amongst others). The different curves follow by solving Eq. (4.34a) for $\zeta^{\prime}=0$ and $B=0$ numerically for different initial slopes. The curves are numbered according to the initial conditions: $\varrho^{\prime}(\xi=0)=(0,-0.5,-0.75,-1.0,-1.5$, $-2.0)$. 
sense in the case that no focusing or defocusing is taken into account. The subsequent two conditions state that an arbitrary initial angle $\varphi_{0}$ is chosen that initially does not change as well. By the fifth condition a head particle is considered and the sixth condition takes the velocity difference $\Delta v$ of this particle with respect to the reference particle into account. Now these initial conditions have to be translated to the dimensionless variables.

The first five can be translated directly by using Eq. (4.18). The last one is a bit more involved. Here we first need the velocity difference $\Delta v$ of the head particle with respect to the reference particle for a bunch traveling through the last bending magnet. In the following, this difference is assumed to be constant. Let $\sigma_{s}^{(4 \text { th })}$ be the bunch length directly before the fourth chicane magnet, $\sigma_{s}^{(\text {fin) }}$ the final bunch length, and $v$ the (constant) velocity of the reference particle. It then makes sense to state that both the head and the tail particle will travel half of the distance $\sigma_{s}^{\text {(fin) }}-\sigma_{s}^{(4 \text { th })}$ during compression. Such a distance will be traveled in the time period $\Delta t=$ $R \arcsin \left(L_{\mathrm{mag}} / R\right) / v$. Then the velocity difference of the head particle with respect to the reference particle can be obtained as follows:

$$
\Delta v=\frac{\sigma_{s}^{(\mathrm{fin})}-\sigma_{s}^{(4 \mathrm{th})}}{2 \Delta t}=\frac{\left(\sigma_{s}^{(\mathrm{fin})}-\sigma_{s}^{(4 \mathrm{th})}\right) v}{2 R \arcsin \left(L_{\mathrm{mag}} / R\right)}<0 .
$$

Now $\Delta v$ has to be expressed via the prefactor in $\dot{z}(t)$ of Eq. (4.19c). This then leads to a dimensionless quantity. Finally we end up with the following dimensionless initial conditions:

$$
\begin{gathered}
\varrho(\xi=0)=1, \quad \varrho^{\prime}(\xi=0)=0, \\
\varphi(\xi=0)=\varphi_{0}, \quad \varphi^{\prime}(\xi=0)=0, \\
\zeta(\xi=0)=1, \\
\zeta^{\prime}(\xi=0)=\Delta v\left(v \sqrt{2 K} \frac{\sigma_{s}^{(4 \text { th })}}{r_{0}}\right)^{-1} .
\end{gathered}
$$

Via Eq. (4.18) the dimensionless variable $\xi$ is related to the dimensionful traveled length $l$. The maximum traveling length $l_{m}$ of the reference particle inside the fourth bending magnet connects to the following $\xi_{m}$ :

$$
\xi_{m} \equiv \sqrt{2 K} \frac{l_{m}}{r_{0}}=\sqrt{2 K} \frac{R \arcsin \left(L_{\mathrm{mag}} / R\right)}{r_{0}} .
$$

The bunch lengths for both bunch charges right before the fourth bending magnet are obtained using the particle trajectory described in Sec. II A. They are corrected by a factor $1 / \cos \alpha$ with the bending angle $\alpha$ since the bunch length obtained with this procedure is understood to be projected on the longitudinal axis. Finally, for the $3 \mathrm{nC}$ bunch we get with the choice $\varphi_{0}=1$ :

$\left.\varrho\left(\xi_{m}\right)\right|_{3 \mathrm{nC}}=1.00956,\left.\quad \varphi\left(\xi_{m}\right)\right|_{3 \mathrm{nC}}=0.997846$,

$\left.\zeta\left(\xi_{m}\right)\right|_{3 \mathrm{nC}}=0.767139$.

The corresponding values for the $1 \mathrm{pC}$ bunch are given by

$\left.\varrho\left(\xi_{m}\right)\right|_{1 \mathrm{pC}}=1.00091,\left.\quad \varphi\left(\xi_{m}\right)\right|_{1 \mathrm{pC}}=0.999135$,

$\left.\zeta\left(\xi_{m}\right)\right|_{1 \mathrm{pC}}=0.704109$.

The dependence of these values on the initial angle was tested as well. For the $1 \mathrm{pC}$ bunch the results vary in the per mill regime, whereas for $3 \mathrm{nC}$ the maximum variations are $2 \%$. Note that the problem is not completely cylindrically symmetric.

How the space charge forces influence bunch compression can be deduced from $\zeta\left(\xi_{m}\right)$. Twice this value corresponds to the amount of bunch compression if it is assumed that the head particle travels the same distance as the tail particle. So we have

$$
\begin{aligned}
\left.\sigma_{s}\right|_{\text {with space charge }} ^{3 \mathrm{nC}} & =\left.\left[\left.2 \zeta\left(\xi_{m}\right)\right|_{3 \mathrm{nC}}-1\right] \sigma_{s}^{(4 \mathrm{th})}\right|_{3 \mathrm{nC}} \\
& =\left.1.069 \sigma_{s}\right|_{3 \mathrm{nC}},
\end{aligned}
$$

for the $3 \mathrm{nC}$ bunch and

$$
\begin{aligned}
\left.\sigma_{s}\right|_{\text {with space charge }} ^{1 \mathrm{pC}} & =\left.\left[\left.2 \zeta\left(\xi_{m}\right)\right|_{1 p C}-1\right] \sigma_{s}^{(4 \mathrm{th})}\right|_{1 \mathrm{pC}} \\
& =\left.1.009 \sigma_{s}\right|_{1 \mathrm{pC}},
\end{aligned}
$$

for the $1 \mathrm{pC}$ bunch. Now we compare these results to the output of the Astra space charge routine that is shown in Fig. 10(a) for the $3 \mathrm{nC}$ bunch and in Fig. 10(b) for the $1 \mathrm{pC}$ bunch. In comparison to the Astra results without space charges the bunch length increases by approximately 5.2\% for $3 \mathrm{nC}$ and $7.5 \%$ or $1 \mathrm{pC}$. Hence the simulation results correspond quite well to the predicted results by the analytical method, which are $6.9 \%$ for $3 \mathrm{nC}$ and $9.5 \%$ for $1 \mathrm{pC}$.

The increase of bunch length caused by space charge forces lies in the regime of few percent for $3 \mathrm{nC}$, which is a factor of around 7 larger than the few per mille for $1 \mathrm{pC}$. The explanation for this is that the product $\psi_{7} \psi_{9} \tilde{\psi}_{11}$ in Table III has a value of 38.0 for $3 \mathrm{nC}$ differing from the corresponding value for $1 \mathrm{pC}$ by a factor of approximately 5.5. Note that the latter product describes the size of the force in the circular magnetic field experienced by an electron moving outwards. This force works against bunch compression. The bunch length of the $1 \mathrm{pC}$ bunch is, indeed, smaller by a factor of 9 versus the $3 \mathrm{nC}$ bunch. However note that the bunch charges differ by a factor of 3000 enlarging the space charge forces for the $3 \mathrm{nC}$ bunch. 


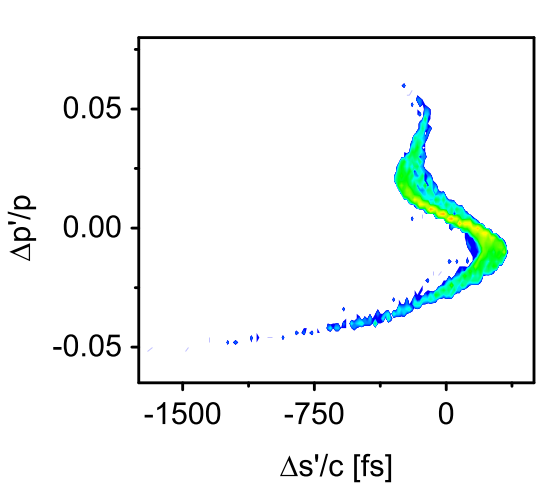

(a)

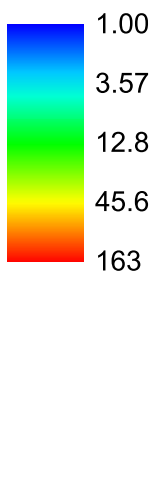

3.57

12.8

163

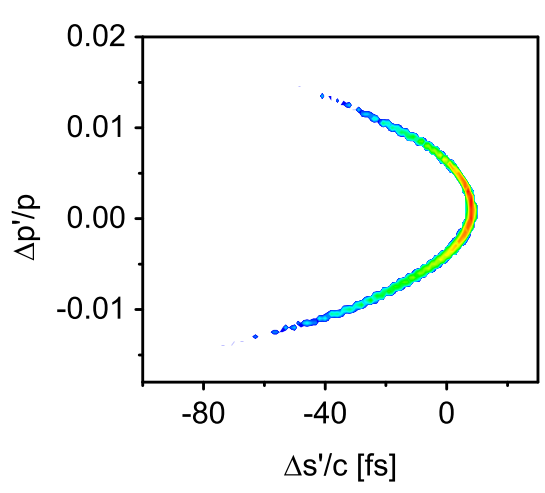

(b)

FIG. 10. Longitudinal phase space plots of tracked $3 \mathrm{nC}$ and $1 \mathrm{pC}$ bunches after the chicane. The profiles shown were computed with the Astra space charge routine based on 5000 macroparticles. The bunch parameters are $Q_{b}=3 \mathrm{nC}, \sigma_{s}=224 \mathrm{fs}$ (a) and $Q_{b}=1 \mathrm{pC}$, $\sigma_{s}=11.5 \mathrm{fs}$ (b).

Finally the space charge simulations for FLUTE performed with Astra shall be directly compared to the analytical approach. Realistic bunches at FLUTE have a complicated structure both in position and momentum space (cf. Figs. 3 and 8). Therefore to test our method directly, ideal cylindrical bunches with charges of $3 \mathrm{nC}$ and $1 \mathrm{pC}$ are initialized in Astra (see Fig. 11 for $3 \mathrm{nC}$ ). As explained in the beginning of the current section, the cylinders are characterized by uniform electron density, sharp edges, and also zero transverse temperatures. Their initial positions are chosen to be $6 \mathrm{~cm}$ before the fourth chicane magnet where they have radii $r_{m}$ and initial lengths $L^{(i)}$ given in Table IV, cf. also Eq. (4.27). Furthermore their correlated energy spreads are set to zero for simplification. Runs without space charge effects lead to final cylinder lengths $L^{(f)}$. With space charge effects the final cylinder lengths are given by $\tilde{L}^{(f)}$ and the explicit values for $L^{(f)}$ and $\tilde{L}^{(f)}$ can be found in Table IV. Those are obtained $6 \mathrm{~cm}$ behind the fourth dipole magnet. Switching space charge effects on, results in an increase of the final cylinder length by around $2.80 \%$ for $3 \mathrm{nC}$ and $5.4 \%$ for $1 \mathrm{pC}$.

Now an analogue computation is carried out using the analytical space charge model. The initial conditions for the set of differential equations are chosen such as in Eq. (4.38)

TABLE IV. Transverse and longitudinal dimensions used for the ideal, cylindrical $3 \mathrm{nC}$ and $1 \mathrm{pC}$ bunches. The initial lengths $L^{(i)}$ are taken $6 \mathrm{~cm}$ before the fourth chicane magnet $(z=11.39 \mathrm{~m})$ and the final lengths $L^{(f)}, \tilde{L}^{(f)}$ are obtained $6 \mathrm{~cm}$ behind the fourth dipole $(z=11.71 \mathrm{~m})$.

\begin{tabular}{lccc}
\hline \hline Parameter & Unit & $Q_{b}=3 \mathrm{nC}$ & $Q_{b}=1 \mathrm{pC}$ \\
\hline$r_{m}$ & $\mathrm{~mm}$ & 2.4 & 0.49 \\
$L^{(i)}$ & $\mathrm{fs}$ & 859.5 & 64.0 \\
$L^{(f)}$ & fs & 879.8 & 71.1 \\
$\tilde{L}^{(f)}$ & $\mathrm{fs}$ & 903.9 & 71.4 \\
\hline \hline
\end{tabular}

with the difference that $\zeta^{\prime}(\xi=0)=0$, since the correlated energy spread of the cylinders is supposed to vanish. Solving the differential equations numerically leads to $\left.\zeta\left(\xi_{m}\right)\right|_{3 \mathrm{nC}}=1.00448$ and $\left.\zeta\left(\xi_{m}\right)\right|_{1 p C}=1.00026$ behind the fourth chicane magnet. Therefore there is an increase of the cylinder length by around $0.9 \%$ for $3 \mathrm{nC}$ and by $0.5 \%$ o for $1 \mathrm{pC}$ accounted for by space charge effects.

Hence the results for the $3 \mathrm{nC}$ and $1 \mathrm{pC}$ bunches obtained from the simple analytical model deviate by factors of approximately 3.1 and 10.8 , respectively, from the simulation results. However it must be respected that it is inherently difficult to model space charge forces in an electron bunch with a complex structure. In the analytical approach the behavior of a single electron is taken as a measure for how the transverse and longitudinal bunch dimensions change where the surrounding charge distribution is assumed to be continuous. This means that effects from individual electrons or clusters of electrons are neglected as well as collective effects. Thus a change of the physical fields originating from fluctuations of charge density is not taken into account. The present study tells us that the model gives a reasonable description of the uniform $3 \mathrm{nC}$ cylinder, but the space charge forces of the $1 \mathrm{pC}$ cylinder are widely underestimated. The cause is most probably a systematic error that is the same for both cylinders, but has much larger implications in the case of a small bunch length such as for the $1 \mathrm{pC}$ bunch (cf. also the discussion at the end of Sec. II A). However the major goal of the analytical model is to understand which particular effects are dominant in increasing the bunch size and which can be neglected. This becomes clear from inspecting the values stated in Table V.

The first term on the right-hand side of Eq. (4.34a) $\left(\sim \eta_{7} \tilde{\eta}_{8}\right)$ involves the generalized perveance $K$, which shows that this contribution is directly linked to space charge forces. It describes that particles are pushed outwards in the radial direction due to space charge effects. The second term $\left(\sim \eta_{7} \eta_{10} \tilde{\eta}_{11}\right)$ originates from the internal 


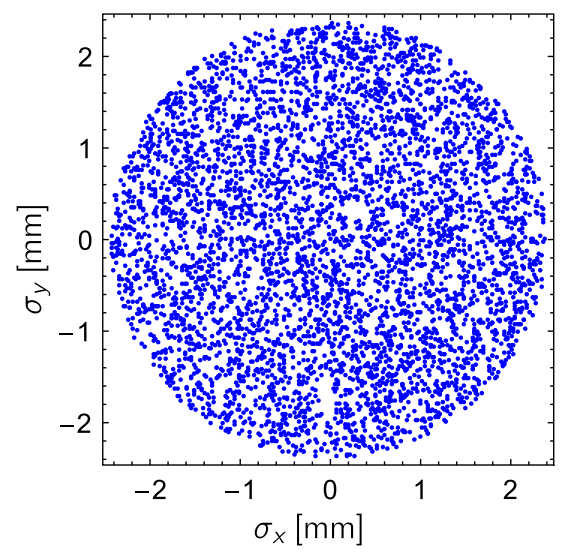

(a)

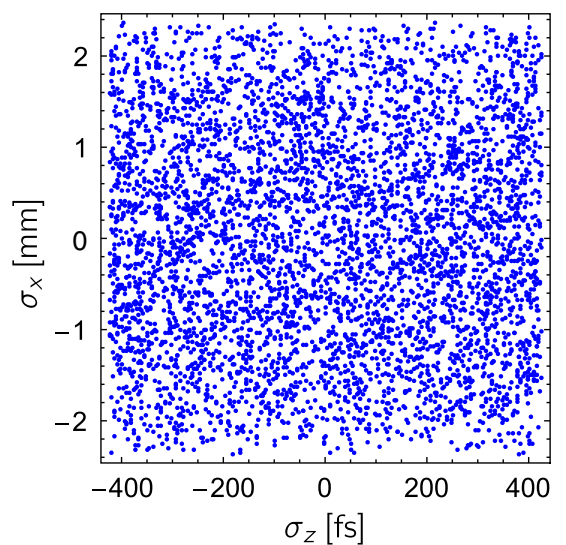

(b)

FIG. 11. Transverse (a) and longitudinal (b) part of the uniform cylindrical test bunch distribution $6 \mathrm{~cm}$ before the fourth dipole for a bunch charge of $3 \mathrm{nC}$. The macroparticles are shown as (blue) dots. The dimensions of the cylinder are chosen according to Eq. (4.27) with the parameters taken from Table II.

magnetic field and it demonstrates that particles driven inwards along the longitudinal direction blow up the bunch radially. Because of the third term $\left(\sim \eta_{7} \eta_{10} B\right)$ the external magnetic field may force particles inwards longitudinally leading to an additional increase of the radial bunch size. The first term on the right-hand side of Eq. (4.34b) $\left(\sim \chi_{7} \chi_{10} B\right)$ indicates that a longitudinal motion of a particle can influence its circular motion. Last but not least, the first two terms on the right-hand side of Eq. (4.34c) $\left(\sim \psi_{7} \psi_{9} \tilde{\psi}_{11}\right.$, $\left.\psi_{7} \psi_{9} B\right)$ involve the generalized perveance and the internal magnetic field, respectively. These contributions again link changes of the radial bunch dimension with changes of the bunch length. The third term $\left(\sim \psi_{7} \psi_{9} B\right)$ reveals that even a circular motion in combination with the external magnetic field may have some influence on the bunch length.

The term governed by $\eta_{7} \eta_{10} \tilde{\eta}_{11}$ in Eq. (4.34a) connects $Q^{\prime \prime}$ and $\zeta^{\prime}$. It describes the Lorentz force acting on an electron when the bunch is being compressed. So a decrease of the longitudinal coordinate is related to an increase in the radial coordinate, which explains the relative minus sign in the differential equation. The term connecting $\zeta^{\prime \prime}$ with $Q^{\prime}$ in Eq. (4.34c) is governed by $\psi_{7} \psi_{9} \tilde{\psi}_{11}$ and it models the Lorentz force on an electron when it is pushed radially outwards. This force leads to an increase in the longitudinal coordinate, which is why the relative sign between the two terms is positive. The ratio of the two forces stated can be calculated to give

TABLE V. Dimensionless physical parameters that appear in the equations of motion (4.24)-(4.26) for the idealized, cylindrical bunches.

\begin{tabular}{ccccccc}
\hline \hline$Q_{b}$ & $\eta_{7} \tilde{\eta}_{8}$ & $\eta_{7} \eta_{10} \tilde{\eta}_{11}$ & $\eta_{7} \eta_{10} B$ & $\chi_{7} \chi_{10} B$ & $\psi_{7} \psi_{9} \tilde{\psi}_{11}$ & $\psi_{7} \psi_{9} B$ \\
\hline $3 \mathrm{nC}$ & 0.500 & 0.436 & 0.205 & 0.205 & 37.8 & 17.8 \\
$1 \mathrm{pC}$ & 0.500 & $1.06 \times 10^{-2}$ & 0.202 & 0.202 & 6.94 & 132 \\
\hline \hline
\end{tabular}

$$
\frac{\zeta^{\prime \prime}}{\varrho^{\prime \prime}}=-\Xi\left(\frac{\varrho^{\prime}}{\zeta^{\prime}}\right), \quad \Xi=\frac{\psi_{7} \psi_{9} \tilde{\psi}_{11}}{\eta_{7} \eta_{10} \tilde{\eta}_{11}}=\left(\frac{r_{0}}{L}\right)^{2} .
$$

Hence the forces themselves are equal in magnitude as expected, since Eq. (4.44) does not involve characteristic quantities associated to the force such as the electron charge or the magnetic flux density. The ratio is solely made up of the radial and longitudinal bunch dimensions $r_{0}$ and $L$, respectively. Note that for the $3 \mathrm{nC}$ bunch $r_{0}$ lies in the order of magnitude of few millimeters, whereas $L$ is a fraction of one millimeter, which explains the quite large difference of the related coefficients in the differential equation. To understand this, assume that an electron propagates outwards in the radial direction along a path length corresponding to a small fraction of the radial bunch dimension. Now if this electron experiences a Lorentz force the resulting deflection along the longitudinal direction will have a much larger impact on the bunch length than the radial motion has on the radial bunch dimension.

To summarize, the results obtained from the analytical approach are in reasonable agreement with the simulations. The predictions for an increase in bunch length for the realistic bunches at FLUTE lie in the right ballpark for both bunches [cf. the paragraph under Eq. (4.43)]. For uniform cylinders of equivalent charges (without a correlated energy spread) the amount of space charge forces is underestimated in the analytical method. This underestimation is compensated when the cylinders are subject to bunch compression caused by a chirp, which leads to the much better agreement for the realistic $3 \mathrm{nC}$ and $1 \mathrm{pC}$ bunches. Nevertheless the analytical approach serves well in giving a rough estimate on space charge forces in a short amount of time (few seconds for solving the Lorentz force equations numerically with Mathematica versus an Astra simulation run including space charge effects taking several hours). Furthermore looking at the equations of motion, the 
physical effects responsible for an increase of the beam size and the bunch length can be identified directly. In addition, a more sophisticated version of our formalism has potential for implementation in tracking codes for substantial reduction of computing power by elongation of step sizes.

\section{CONCLUSIONS AND OUTLOOK}

To summarize, analytical studies for bunch compression at the future linear accelerator FLUTE were performed whose results were compared to the simulation output of the tool Astra. The calculations were done for two typical bunches with the extremal charges of $1 \mathrm{pC}$ and $3 \mathrm{nC}$ that had been simulated from the cathode to the entrance of the bunch compressor. Neglecting both space charge and CSR effects, the final bunch profiles obtained from mere path length differences agree very well with the simulation results. As a cross-check, the problem was then treated within the transfer matrix formalism as well. First-order perturbation theory in the momentum deviation gives a result for the final bunch length that deviates from the simulation results by several percent. For this reason and because of dispersive effects considering second-order terms was mandatory to give a good agreement. Besides, in this context we obtained some new first- and secondorder transfer coefficients for dipole magnets, fringe fields, and drifts.

To consider space charge effects, a simple model was introduced where the bunch is described by a homogeneously charged cylinder. The latter generates both electric and magnetic fields when moving. The equations of motion for a single electron at the surface of the cylinder were obtained and solved numerically. For realistic bunches at FLUTE this model gives a reasonable agreement of the increase in bunch length with the simulations. It can be deduced that space charge effects are negligible for bunch compression at FLUTE. Our approach is simple enough to allow "back-on-the-envelope" estimations on both transverse and longitudinal space charge forces for an electron bunch on a curved trajectory without the need of timeconsuming simulations.

The paper demonstrates how powerful the combination of analytical methods and simulations is to investigate bunch compression. The techniques presented shall provide a framework for further analytical compression studies. These can be used for future investigations of FLUTE or they may be modified accordingly for other purposes.

\section{ACKNOWLEDGMENTS}

It is a pleasure to thank M. Fitterer, S. Hillenbrand, V. Judin, A.-S. Müller, S. Marsching, M. Nasse, A. Papash, R. Rossmanith, M. Schuh, and M. Weber for helpful discussions. We thank S. Naknaimueang for useful discussions, reading the paper, and for updating some of the bunch profile plots with Origin. Furthermore the authors are indebted to M. Oyamada and M. Schwarz for reading the paper and giving helpful comments. Furthermore we are indebted to the two anonymous referees for useful suggestions improving the manuscript. This work was mainly performed with financial support within the program "Accelerator Research and Development" of the Hermann von Helmholtz-Gemeinschaft Deutscher Forschungszentren e.V. One of us (M.S.) acknowledges additional support from the Deutsche Akademie der Naturforscher Leopoldina within Grant No. LPDS 201217 to complete this paper.

\section{APPENDIX A: PARAMETRIC REPRESENTATION OF PARTICLE TRAJECTORY IN THE FLUTE CHICANE}

In what follows, find the electron trajectory for the FLUTE chicane used in Sec. II A (for initial angle $x^{\prime}=0$ ), cf. Fig. 12. The right-hand interval limits of $\mathbf{r}_{1}, \ldots, \mathbf{r}_{9}$ are understood to correspond to $l_{1}, \ldots, l_{9}$ :

$$
\begin{aligned}
& \mathbf{r}(l)= \begin{cases}\mathbf{r}_{1}(l) & \text { for } 0 \leq l \leq l_{1}, \\
\mathbf{r}_{2}\left(l-l_{1}\right) & \text { for } l_{1} \leq l \leq \sum_{i=1}^{2} l_{i}, \\
\mathbf{r}_{3}\left(l-\sum_{i=1}^{2} l_{i}\right) & \text { for } \sum_{i=1}^{2} l_{i} \leq l \leq \sum_{i=1}^{3} l_{i}, \\
\mathbf{r}_{4}\left(l-\sum_{i=1}^{3} l_{i}\right) & \text { for } \sum_{i=1}^{3} l_{i} \leq l \leq \sum_{i=1}^{4} l_{i}, \\
\mathbf{r}_{5}\left(l-\sum_{i=1}^{4} l_{i}\right) & \text { for } \sum_{i=1}^{4} l_{i} \leq l \leq \sum_{i=1}^{5} l_{i}, \\
\mathbf{r}_{6}\left(l-\sum_{i=1}^{5} l_{i}\right) & \text { for } \sum_{i=1}^{5} l_{i} \leq l \leq \sum_{i=1}^{6} l_{i}, \\
\mathbf{r}_{7}\left(l-\sum_{i=1}^{6} l_{i}\right) & \text { for } \sum_{i=1}^{6} l_{i} \leq l \leq \sum_{i=1}^{7} l_{i}, \\
\mathbf{r}_{8}\left(l-\sum_{i=1}^{7} l_{i}\right) & \text { for } \sum_{i=1}^{7} l_{i} \leq l \leq \sum_{i=1}^{8} l_{i}, \\
\mathbf{r}_{9}\left(l-\sum_{i=1}^{8} l_{i}\right) & \text { for } \sum_{i=1}^{8} l_{i} \leq l \leq \sum_{i=1}^{9} l_{i},\end{cases} \\
& \mathbf{r}_{1}(l)=\left(\begin{array}{c}
x_{0} \\
z_{0}
\end{array}\right)+\left(\begin{array}{c}
\sin x^{\prime} \\
\cos x^{\prime}
\end{array}\right) l, \quad l \in\left[0, \frac{z_{1}-z_{0}}{\cos x^{\prime}}\right], \\
& \mathbf{r}_{2}(l)=\mathbf{r}_{1}\left(\frac{z_{1}-z_{0}}{\cos x^{\prime}}\right)+R\left(\begin{array}{c}
\cos x^{\prime}-\cos \left(l / R+x^{\prime}\right) \\
\sin \left(l / R+x^{\prime}\right)-\sin x^{\prime}
\end{array}\right), \\
& l \in[0, R \alpha], \\
& \mathbf{r}_{3}(l)=\mathbf{r}_{2}(R \alpha)+\left(\begin{array}{c}
\cos \delta \\
\sin \delta
\end{array}\right) l, \quad l \in\left[0, \frac{L_{\text {space }}}{\sin \delta}\right], \\
& \mathbf{r}_{4}(l)=\mathbf{r}_{3}\left(\frac{L_{\text {space }}}{\sin \delta}\right)+R\left(\begin{array}{c}
\sin (l / R+\delta)-\sin \delta \\
\cos \delta-\cos (l / R+\delta)
\end{array}\right), \\
& l \in[0, R \alpha], \\
& \mathbf{r}_{5}(l)=\mathbf{r}_{4}(R \alpha)+\left(\begin{array}{c}
\sin x^{\prime} \\
\cos x^{\prime}
\end{array}\right) l, \quad l \in\left[0, \frac{L_{\mathrm{drift}}}{\cos x^{\prime}}\right],
\end{aligned}
$$




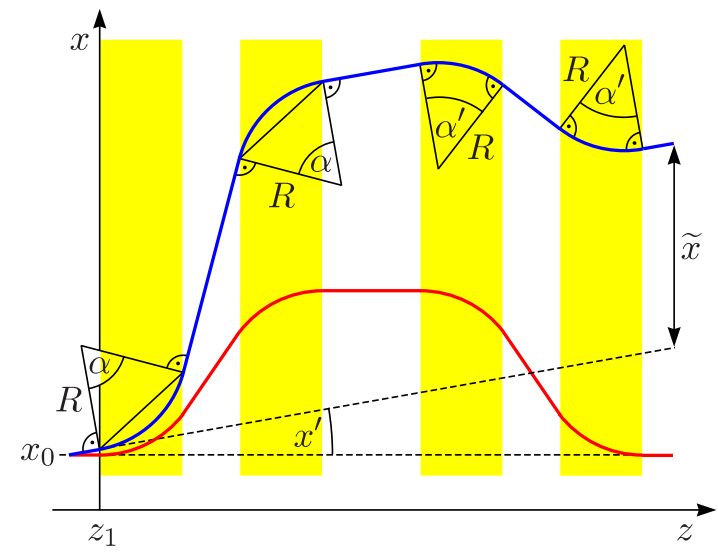

FIG. 12. Here the FLUTE chicane is considered with a particle trajectory traveled by an electron that has both an initial transverse momentum and a longitudinal momentum component. Hence the angle $x^{\prime}$ between the initial straight trajectory and the horizontal axis is nonzero. The resulting modified trajectory is shown as a plain, blue curve. The trajectory for $x^{\prime}=0$ is shown as a plain, red curve and it is presented for comparison. The electron after the chicane does not come back to the $z$-axis for initial $x^{\prime} \neq 0$.

$$
\begin{aligned}
\mathbf{r}_{6}(l)= & \mathbf{r}_{5}\left(\frac{L_{\mathrm{drift}}}{\cos x^{\prime}}\right)+R\left(\begin{array}{c}
\cos \left(l / R-x^{\prime}\right)-\cos x^{\prime} \\
\sin \left(l / R-x^{\prime}\right)+\sin x^{\prime}
\end{array}\right), \\
& l \in\left[0, R\left(x^{\prime}+\epsilon\right)\right], \\
\mathbf{r}_{7}(l)= & \mathbf{r}_{6}\left(R\left[x^{\prime}+\epsilon\right]\right)+\left(\begin{array}{c}
-\sin \epsilon \\
\cos \epsilon
\end{array}\right) l, \quad l \in\left[0, \frac{L_{\text {space }}}{\cos \epsilon}\right],
\end{aligned}
$$

$$
\begin{gathered}
\mathbf{r}_{8}(l)=\mathbf{r}_{7}\left(\frac{L_{\text {space }}}{\cos \epsilon}\right)+R\left(\begin{array}{c}
\cos \epsilon-\cos (\epsilon-l / R) \\
\sin \epsilon-\sin (\epsilon-l / R)
\end{array}\right), \\
l \in\left[0, R\left(x^{\prime}+\epsilon\right)\right],
\end{gathered}
$$

$$
\mathbf{r}_{9}(l)=\mathbf{r}_{8}\left(R\left[x^{\prime}+\epsilon\right]\right)+\left(\begin{array}{c}
\sin x^{\prime} \\
\cos x^{\prime}
\end{array}\right) l, \quad l \in\left[0, z_{2}\right]
$$

$$
\begin{aligned}
& \alpha=\arcsin \left(\frac{L_{\mathrm{mag}}}{R}+\sin x^{\prime}\right)-x^{\prime}, \\
& \delta=\frac{\pi}{2}-\left(\alpha+x^{\prime}\right), \\
& \epsilon=\arcsin \left(\frac{L_{\mathrm{mag}}}{R}-\sin x^{\prime}\right) .
\end{aligned}
$$

\section{Parametric representation of trajectory in hypothetical sector magnet chicane}

A parametric representation of the trajectory for a particle in a chicane consisting of sector dipole magnets (see Sec. II B and Fig. 13) is given by Eq. (A1a) with the following piecewise functions:

$$
\begin{aligned}
& \mathbf{r}_{1}(l)=\left(\begin{array}{c}
x_{0} \\
z_{0}+l
\end{array}\right), \quad l \in\left[0, z_{1}-z_{0}\right] \\
& \mathbf{r}_{2}(l)=\mathbf{r}_{1}\left(z_{1}-z_{0}\right)+\left(\begin{array}{c}
R \\
0
\end{array}\right)+R^{\prime}\left(\begin{array}{c}
-\cos \left(l / R^{\prime}\right) \\
\sin \left(l / R^{\prime}\right)
\end{array}\right), \\
& l \in\left[0, R^{\prime} \varepsilon\right], \\
& \mathbf{r}_{3}(l)=\mathbf{r}_{2}\left(R^{\prime} \varepsilon\right)+\left(\begin{array}{c}
\sin \varepsilon \\
\cos \varepsilon
\end{array}\right) l, \quad l \in\left[0, L_{\text {space }}^{\prime}\right], \\
& \mathbf{r}_{4}(l)=\mathbf{r}_{3}\left(L_{\text {space }}^{\prime}\right)+R^{\prime \prime}\left(\begin{array}{c}
\cos \left(l / R^{\prime \prime}-\varepsilon\right)-\cos \varepsilon \\
\sin \left(l / R^{\prime \prime}-\varepsilon\right)+\sin \varepsilon
\end{array}\right), \\
& l \in\left[0, R^{\prime \prime} \varepsilon\right], \\
& \mathbf{r}_{5}(l)=\mathbf{r}_{4}\left(R^{\prime \prime} \varepsilon\right)+\left(\begin{array}{l}
0 \\
l
\end{array}\right), \quad l \in\left[0, L_{\mathrm{drift}}\right], \\
& \mathbf{r}_{6}(l)=\mathbf{r}_{5}\left(L_{\text {drift }}\right)+R^{\prime \prime}\left(\begin{array}{c}
\cos \left(l / R^{\prime \prime}\right)-1 \\
\sin \left(l / R^{\prime \prime}\right)
\end{array}\right), \\
& l \in\left[0, R^{\prime \prime} \varepsilon\right], \\
& \mathbf{r}_{7}(l)=\mathbf{r}_{6}\left(R^{\prime \prime} \varepsilon\right)+\left(\begin{array}{c}
-\sin \varepsilon \\
\cos \varepsilon
\end{array}\right) l, \\
& l \in\left[0, L_{\text {space }}^{\prime}\right] \text {, } \\
& \mathbf{r}_{8}(l)=\mathbf{r}_{7}\left(L_{\text {space }}^{\prime}\right)+R^{\prime}\left(\begin{array}{c}
\cos \varepsilon-\cos \left(l / R^{\prime}-\varepsilon\right) \\
\sin \varepsilon+\sin \left(l / R^{\prime}-\varepsilon\right)
\end{array}\right), \\
& l \in\left[0, R^{\prime} \varepsilon\right], \\
& \mathbf{r}_{9}(l)=\mathbf{r}_{8}\left(R^{\prime} \varepsilon\right)+\left(\begin{array}{l}
0 \\
l
\end{array}\right), \quad l \in\left[0, z_{2}-z_{1}\right] \\
& R^{\prime}=R+\Delta R \text {, }
\end{aligned}
$$

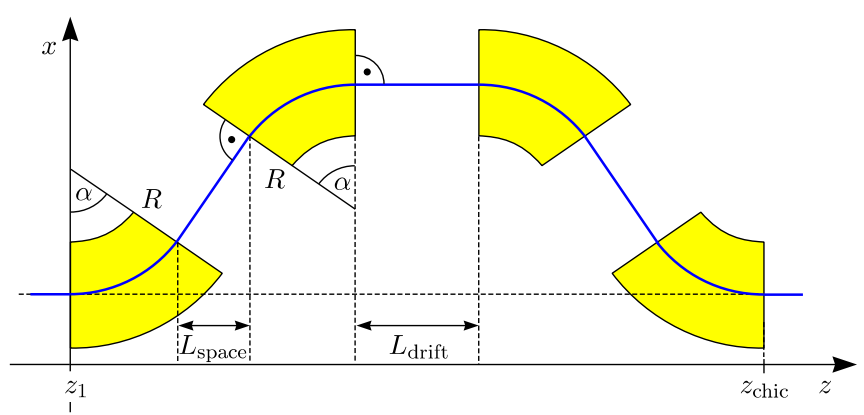

FIG. 13. Draft of a bunch compressor consisting of sector dipole magnets. The reference trajectory is shown as a plain, blue line. The bending angle is given by $\alpha$ and the bending radius is denoted as $R$. The distance between the exit face of the first/third dipole magnet and the entrance face of the second/fourth dipole magnet that is projected on the longitudinal axis is called $L_{\text {space }}$. The distance between the second and the third magnet is $L_{\text {drift }}$. 


$$
\begin{gathered}
\varepsilon=\arctan \left\{\frac{R \sin \alpha+\sin \alpha\left[\sqrt{R^{\prime 2}-\Delta R^{2} \sin ^{2} \alpha}-(R+\Delta R \cos \alpha)\right]}{\Delta R+R \cos \alpha+\cos \alpha\left[\sqrt{R^{\prime 2}-\Delta R^{2} \sin ^{2} \alpha}-(R+\Delta R \cos \alpha)\right]}\right\}, \\
L_{\text {space }}^{\prime}=\frac{2 L_{\text {space }}-\Delta R \sin (2 \alpha)-2 R^{\prime} \sin \varepsilon}{2 \cos \alpha \cos (\alpha-\varepsilon)}+R^{\prime} \tan \alpha, \\
R^{\prime \prime}=\frac{1}{\cos (\alpha-\varepsilon)}\left\{R[\sin (2 \alpha)+\cot \varepsilon] \tan (\alpha)-R \cot \varepsilon \sin ^{2} \alpha \tan \alpha\right. \\
\left.+\sin \alpha\left[L_{\text {space }}-R^{\prime} / \sin \varepsilon+\cot \varepsilon\left(R^{\prime}-R+R \cos \alpha-L_{\text {space }} \tan \alpha\right)\right]\right\} .
\end{gathered}
$$

Here $\alpha$ is the bending angle and $\Delta R=\Delta p /(e B)$ with the momentum deviation $\Delta p$, the magnetic field $B$, and the elementary charge $e$. For the reference trajectory $\Delta R=0$ has to be set.

\section{APPENDIX B: SPACE CHARGE EFFECTS FOR A CYLINDRIC BUNCH}

To derive the equations of motion for electrons within a cylindric bunch in Sec. IV the following formulas are needed. The basis vectors $\hat{\mathbf{b}}$ and $\hat{\mathbf{n}}$ can be expressed by the new basis vectors $\hat{\mathbf{e}}_{r}$ and $\hat{\mathbf{e}}_{\vartheta}$ (and vice versa) as follows:

$$
\begin{aligned}
& \hat{\mathbf{e}}_{r}=\hat{\mathbf{n}} \cos \vartheta+\hat{\mathbf{b}} \sin \vartheta, \\
& \hat{\mathbf{e}}_{\vartheta}=-\hat{\mathbf{n}} \sin \vartheta+\hat{\mathbf{b}} \cos \vartheta, \\
& \hat{\mathbf{b}}=\hat{\mathbf{e}}_{r} \sin \vartheta+\hat{\mathbf{e}}_{\vartheta} \cos \vartheta, \\
& \hat{\mathbf{n}}=\hat{\mathbf{e}}_{r} \cos \vartheta-\hat{\mathbf{e}}_{\vartheta} \sin \vartheta .
\end{aligned}
$$

The derivatives of the basis vectors $\left\{\hat{\mathbf{e}}_{r}, \hat{\mathbf{e}}_{\vartheta}, \hat{\mathbf{t}}\right\}$ with respect to $t$ are given by

$$
\begin{aligned}
\dot{\hat{\mathbf{e}}}_{r}= & -\hat{\mathbf{n}} \dot{\vartheta} \sin \vartheta+\cos \vartheta|\dot{\mathbf{r}}|(\hat{\mathbf{b}} \tau-\hat{\mathbf{t}} \kappa)+\hat{\mathbf{b}} \dot{\vartheta} \cos \vartheta \\
& +\sin \vartheta(-\hat{\mathbf{n}}|\dot{\mathbf{r}}| \tau) \\
= & -\hat{\mathbf{t}}|\dot{\mathbf{r}}| \kappa \cos \vartheta+\dot{\vartheta}(-\hat{\mathbf{n}} \sin \vartheta+\hat{\mathbf{b}} \cos \vartheta) \\
& +|\dot{\mathbf{r}}| \tau(\hat{\mathbf{b}} \cos \vartheta-\hat{\mathbf{n}} \sin \vartheta) \\
= & -\hat{\mathbf{t}}|\dot{\mathbf{r}}| \kappa \cos \vartheta+(\dot{\vartheta}+|\dot{\mathbf{r}}| \tau) \hat{\mathbf{e}}_{\vartheta}, \quad(\mathrm{B} 2 \mathrm{a}) \\
\dot{\hat{\mathbf{e}}}_{\vartheta}= & -\hat{\mathbf{n}} \dot{\vartheta} \cos \vartheta-\sin \vartheta|\dot{\mathbf{r}}|(\hat{\mathbf{b}} \tau-\hat{\mathbf{t}} \kappa)-\hat{\mathbf{b}} \dot{\vartheta} \sin \vartheta+\dot{\hat{\mathbf{b}} \cos \vartheta} \\
= & \hat{\mathbf{t}}|\dot{\mathbf{r}}| \kappa \sin \vartheta-\dot{\vartheta}(\hat{\mathbf{n}} \cos \vartheta+\hat{\mathbf{b}} \sin \vartheta) \\
& -|\dot{\mathbf{r}}| \tau(\hat{\mathbf{n}} \cos \vartheta+\hat{\mathbf{b}} \sin \vartheta) \\
= & \hat{\mathbf{t}}|\dot{\mathbf{r}}| \kappa \sin \vartheta-\hat{\mathbf{e}}_{r}(\dot{\vartheta}+|\dot{\mathbf{r}}| \tau),
\end{aligned}
$$

$$
\dot{\hat{\mathbf{t}}}=\hat{\mathbf{n}}|\dot{\mathbf{r}}| \kappa=\hat{\mathbf{e}}_{r}|\dot{\mathbf{r}}| \kappa \cos \vartheta-\hat{\mathbf{e}}_{\vartheta}|\dot{\mathbf{r}}| \kappa \sin \vartheta
$$

$$
\begin{aligned}
\ddot{\hat{\mathbf{e}}}_{r}= & -\hat{\mathbf{t}}\left(\frac{\mathrm{d}|\dot{\mathbf{r}}|}{\mathrm{d} t} \kappa \cos \vartheta+|\dot{\mathbf{r}}| \dot{\kappa} \cos \vartheta-\dot{\vartheta}|\dot{\mathbf{r}}| \kappa \sin \vartheta\right) \\
& -\dot{\hat{\mathbf{t}}}|\dot{\mathbf{r}}| \kappa \cos \vartheta+\hat{\mathbf{e}}_{\vartheta}\left(\ddot{\vartheta}+\frac{\mathrm{d}|\dot{\mathbf{r}}|}{\mathrm{d} t} \tau+|\dot{\mathbf{r}}| \dot{\tau}\right)+\dot{\hat{\mathbf{e}}}_{\vartheta}(\dot{\vartheta}+|\dot{\mathbf{r}}| \tau) \\
= & -\hat{\mathbf{t}}\left(\frac{\mathrm{d}|\dot{\mathbf{r}}|}{\mathrm{d} t} \kappa \cos \vartheta+|\dot{\mathbf{r}}| \dot{\kappa} \cos \vartheta-2 \dot{\vartheta}|\dot{\mathbf{r}}| \kappa \sin \vartheta-|\dot{\mathbf{r}}|^{2} \tau \kappa \sin \vartheta\right) \\
& +\hat{\mathbf{e}}_{\vartheta}\left(\ddot{\vartheta}+\frac{\mathrm{d}|\dot{\mathbf{r}}|}{\mathrm{d} t} \tau+|\dot{\mathbf{r}}| \dot{\tau}+|\dot{\mathbf{r}}|^{2} \kappa^{2} \sin \vartheta \cos \vartheta\right) \\
& -\hat{\mathbf{e}}_{r}\left[(\dot{\vartheta}+|\dot{\mathbf{r}}| \tau)^{2}+|\dot{\mathbf{r}}|^{2} \kappa^{2} \cos ^{2} \vartheta\right], \quad(\mathrm{B} 2 \mathrm{~d})
\end{aligned}
$$

$$
\begin{aligned}
\ddot{\hat{\mathbf{t}}}= & \hat{\mathbf{e}}_{r}\left(\frac{\mathrm{d}|\dot{\mathbf{r}}|}{\mathrm{d} t} \kappa \cos \vartheta+|\dot{\mathbf{r}}| \dot{\kappa} \cos \vartheta-|\dot{\mathbf{r}}| \kappa \dot{\vartheta} \sin \vartheta\right)+\dot{\hat{\mathbf{e}}}_{r}|\dot{\mathbf{r}}| \kappa \cos \vartheta \\
& -\hat{\mathbf{e}}_{\vartheta}\left(\frac{\mathrm{d}|\dot{\mathbf{r}}|}{\mathrm{d} t} \kappa \sin \vartheta+|\dot{\mathbf{r}}| \dot{\kappa} \sin \vartheta+|\dot{\mathbf{r}}| \kappa \dot{\vartheta} \cos \vartheta\right)-\dot{\hat{\mathbf{e}}}_{\vartheta}|\dot{\mathbf{r}}| \kappa \sin \vartheta
\end{aligned}
$$$$
=\hat{\mathbf{e}}_{r}\left(\frac{\mathrm{d}|\dot{\mathbf{r}}|}{\mathrm{d} t} \kappa \cos \vartheta+|\dot{\mathbf{r}}| \dot{\kappa} \cos \vartheta-|\dot{\mathbf{r}}| \kappa \dot{\vartheta} \sin \vartheta\right.
$$$$
+(\dot{\vartheta}+|\dot{\mathbf{r}}| \tau)|\dot{\mathbf{r}}| \kappa \sin \vartheta)
$$$$
-\hat{\mathbf{e}}_{\vartheta}\left[\frac{\mathrm{d}|\dot{\mathbf{r}}|}{\mathrm{d} t} \kappa \sin \vartheta+|\dot{\mathbf{r}}| \dot{\kappa} \sin \vartheta+|\dot{\mathbf{r}}| \kappa \dot{\vartheta} \cos \vartheta\right.
$$$$
-|\dot{\mathbf{r}}| \kappa \cos \vartheta(\dot{\vartheta}+|\dot{\mathbf{r}}| \tau)]-\hat{\mathbf{t}}|\dot{\mathbf{r}}|^{2} \kappa^{2}
$$

From these results the velocity and the acceleration vector that are used in Eq. (4.12) can be computed:

$$
\begin{aligned}
\dot{\mathbf{r}}_{b}= & \dot{r} \hat{\mathbf{e}}_{r}+r \dot{\hat{\mathbf{e}}}_{r}+\dot{z} \hat{\mathbf{t}}+z \dot{\hat{\mathbf{t}}} \\
= & \hat{\mathbf{t}}(\dot{z}-r|\dot{\mathbf{r}}| \kappa \cos \vartheta)+\hat{\mathbf{e}}_{\vartheta}[r(\dot{\vartheta}+|\dot{\mathbf{r}}| \tau)-z|\dot{\mathbf{r}}| \kappa \sin \vartheta] \\
& +\hat{\mathbf{e}}_{r}(\dot{r}+z|\dot{\mathbf{r}}| \kappa \cos \vartheta),
\end{aligned}
$$




$$
\begin{aligned}
\ddot{\mathbf{r}}_{b}= & \ddot{r} \hat{\mathbf{e}}_{r}+2 \dot{\dot{r}} \dot{\hat{\mathbf{e}}}_{r}+r \ddot{\hat{\mathbf{e}}}_{r}+\ddot{z} \hat{\mathbf{t}}+2 \dot{\bar{z}} \dot{\hat{\mathbf{t}}}+z \ddot{\hat{\mathbf{t}}} \\
= & \hat{\mathbf{t}}\left[-r\left(\frac{\mathrm{d}|\dot{\mathbf{r}}|}{\mathrm{d} t} \kappa \cos \vartheta+|\dot{\mathbf{r}}| \dot{\kappa} \cos \vartheta-2 \dot{\vartheta}|\dot{\mathbf{r}}| \kappa \sin \vartheta-|\dot{\mathbf{r}}|^{2} \tau \kappa \sin \vartheta\right)+\ddot{z}-|\dot{\mathbf{r}}|^{2} \kappa^{2} z-2 \dot{r}|\dot{\mathbf{r}}| \kappa \cos \vartheta\right] \\
& +\hat{\mathbf{e}}_{\vartheta}\left[r\left(\ddot{\vartheta}+\frac{\mathrm{d}|\dot{\mathbf{r}}|}{\mathrm{d} t} \tau+|\dot{\mathbf{r}}| \dot{\tau}+|\dot{\mathbf{r}}|^{2} \kappa^{2} \sin \vartheta \cos \vartheta\right)-2 \dot{z}|\dot{\mathbf{r}}| \kappa \sin \vartheta\right. \\
& \left.-z\left(\frac{\mathrm{d}|\dot{\mathbf{r}}|}{\mathrm{d} t} \kappa \sin \vartheta+|\dot{\mathbf{r}}| \dot{\kappa} \sin \vartheta-|\dot{\mathbf{r}}|^{2} \kappa \tau \cos \vartheta\right)+2 \dot{r}(\dot{\vartheta}+|\dot{\mathbf{r}}| \tau)\right] \\
& +\hat{\mathbf{e}}_{r}\left[-r\left((\dot{\vartheta}+|\dot{\mathbf{r}}| \tau)^{2}+|\dot{\mathbf{r}}|^{2} \kappa^{2} \cos ^{2} \vartheta\right)+2 \dot{z}|\dot{\mathbf{r}}| \kappa \cos \vartheta+z\left(\frac{\mathrm{d}|\dot{\mathbf{r}}|}{\mathrm{d} t} \kappa \cos \vartheta+|\dot{\mathbf{r}}| \dot{\kappa} \cos \vartheta+|\dot{\mathbf{r}}|^{2} \kappa \tau \sin \vartheta\right)+\ddot{r}\right] .
\end{aligned}
$$

Finally, the following cross product is needed to obtain the Lorentz force:

$$
\begin{aligned}
\dot{\mathbf{r}} \times \mathbf{B}= & \left(\dot{\mathbf{r}}_{r} \hat{\mathbf{e}}_{r}+\dot{\mathbf{r}}_{\vartheta} \hat{\mathbf{e}}_{\vartheta}+\dot{\mathbf{r}}_{t} \hat{\mathbf{t}}\right) \times\left(B_{r} \hat{\mathbf{e}}_{r}+B_{\vartheta} \hat{\mathbf{e}}_{\vartheta}+B_{t} \hat{\mathbf{t}}\right) \\
= & \left(\dot{\mathbf{r}}_{r} B_{\vartheta}-\dot{\mathbf{r}}_{\vartheta} B_{r}\right) \hat{\mathbf{t}}+\left(\dot{\mathbf{r}}_{t} B_{r}-\dot{\mathbf{r}}_{r} B_{t}\right) \hat{\mathbf{e}}_{\vartheta} \\
& +\left(\dot{\mathbf{r}}_{\vartheta} B_{t}-\dot{\mathbf{r}}_{t} B_{\vartheta}\right) \hat{\mathbf{e}}_{r} .
\end{aligned}
$$

The general differential equations are given below together with the remaining coefficients that are not needed in Sec. IV:

$$
\begin{aligned}
& \eta_{1}\left(\varrho^{\prime}+\eta_{2} f \zeta \cos \varphi\right)+\varrho^{\prime \prime}+\eta_{3} \varrho^{\prime} \\
& +\left[\eta_{4}\left(\eta_{3} f+g\right) \zeta+\eta_{5} f \zeta^{\prime}\right] \cos \varphi-\varrho\left(\varphi^{\prime 2}+\eta_{6} f^{2} \cos ^{2} \varphi\right) \\
& =\eta_{7}\left[-\eta_{8}\left(\tilde{E}_{\varrho}^{\mathrm{int}}-\tilde{v} \tilde{B}_{\varphi}^{\mathrm{int}}\right)-\left(\eta_{9} f \varrho \cos \varphi-\eta_{10} \zeta^{\prime}\right)\left(\tilde{B}_{\varphi}^{\mathrm{int}}+\tilde{B}_{\varphi}^{\mathrm{ext}}\right)\right. \\
& \left.-\left(\eta_{12} \varrho \varphi^{\prime}-\eta_{13} f \zeta \sin \varphi\right) \tilde{B}_{t}^{\text {int }}\right] \text {, } \\
& \eta_{8}=\frac{1}{r_{0}}, \quad \eta_{12}=\frac{\sqrt{2 K} \tilde{v}}{r_{0}}, \quad \eta_{13}=\frac{\sqrt{2 K} \tilde{v}}{r_{0}} \frac{L}{R} \\
& \chi_{1}\left(\varrho \varphi^{\prime}-\chi_{2} f \zeta \sin \varphi\right)+\varrho\left[\varphi^{\prime \prime}+\chi_{3} \varphi^{\prime}+\chi_{4} f^{2} \sin (2 \varphi)\right] \\
& +2 \varrho^{\prime} \varphi^{\prime}-\left[\chi_{5}\left(\chi_{3} f+g\right) \zeta+\chi_{6} f \zeta^{\prime}\right] \sin \varphi \\
& =\chi_{7}\left[-\chi_{8}\left(\tilde{E}_{\varphi}^{\mathrm{int}}+\tilde{v} \tilde{B}_{\varrho}^{\mathrm{int}}\right)+\left(\chi_{9} f \varrho \cos \varphi-\chi_{10} \zeta^{\prime}\right)\left(\tilde{B}_{\varrho}^{\mathrm{int}}+\tilde{B}_{\varrho}^{\mathrm{ext}}\right)\right. \\
& \left.+\left(\chi_{11} f \zeta \cos \varphi+\chi_{12} \varrho^{\prime}\right) \tilde{B}_{t}^{\mathrm{int}}\right] \text {, } \\
& \chi_{8}=\frac{1}{r_{0}}, \quad \chi_{11}=\frac{\sqrt{2 K} \tilde{v}}{r_{0}} \frac{L}{R}, \quad \chi_{12}=\frac{\sqrt{2 K} \tilde{v}}{r_{0}} .
\end{aligned}
$$

$$
\begin{aligned}
\psi_{1}\left(\zeta^{\prime}\right. & \left.-\psi_{2} f \varrho \cos \varphi\right)+\zeta^{\prime \prime}+\psi_{3} \zeta^{\prime}-\psi_{4} f^{2} \zeta-\psi_{5} f \varrho^{\prime} \cos \varphi \\
& +\varrho\left[\psi_{5} f \varphi^{\prime} \sin \varphi-\psi_{6}\left(\psi_{3} f+g\right) \cos \varphi\right] \\
= & \psi_{7}\left[-\psi_{8} \tilde{E}_{t}^{\mathrm{int}}-\left(\psi_{9} \rho^{\prime}+\psi_{10} f \zeta \cos \varphi\right)\left(\tilde{B}_{\varphi}^{\mathrm{int}}+\tilde{B}_{\varphi}^{\mathrm{ext}}\right)\right. \\
& \left.+\left(\psi_{9} \rho \varphi^{\prime}-\psi_{10} f \zeta \sin \varphi\right)\left(\tilde{B}_{\varrho}^{\mathrm{int}}+\tilde{B}_{\varrho}^{\mathrm{ext}}\right)\right], \quad(\mathrm{B} 7 \mathrm{a})
\end{aligned}
$$

$$
\psi_{8}=\frac{1}{L}
$$

[1] S. Naknaimueang et al., FLUTE, a linac based THz source, in Proceedings of the 2nd International Particle Accelerator Conference, San Sebastián, Spain (EPS-AG, Geneva, Switzerland, 2011), p. 1458.

[2] S. Naknaimueang et al., FLUTE, a compact acceleratorbased source for coherent $\mathrm{THz}$ radiation, in Proceedings of the 34th International Free-Electron Laser Conference, Nara, Japan, 2012 (JACoW, Geneva, Switzerland, 2013), p. 511.

[3] S. Naknaimueang et al., Optimization of the beam optical parameters of the linac-based terahertz source FLUTE, in Proceedings of the 3rd International Particle Accelerator Conference, New Orleans, LA, 2012 (IEEE, Piscataway, NJ, 2012), p. 1629.

[4] M. Schwarz et al., Comparison of various sources of coherent THz radiation at FLUTE, in Proceedings of the 3rd International Particle Accelerator Conference, New Orleans, LA, 2012 (Ref. [3]), p. 568.

[5] M. Nasse et al., FLUTE: A versatile linac-based $\mathrm{THz}$ source, Rev. Sci. Instrum. 84, 022705 (2013).

[6] R. Assmann et al., FLUTE-A linac-based THz source, Conceptual Design Report, edited by M. Schwarz. (unpublished).

[7] S. Naknaimueang et al., Design studies for FLUTE, a linac-based source of terahertz radiation, in Proceedings of the 35th International Free-Electron Laser Conference, New York, 2013 (JACoW, Geneva, Switzerland, 2013), p. 598.

[8] S. Naknaimueang et al., Simulating the bunch structure in the THz source FLUTE, in Proceedings of the 4th International Particle Accelerator Conference, IPAC2013, Shanghai, China, 2013 (JACoW, Geneva, Switzerland, 2013), p. 2141.

[9] M. Nasse et al., FLUTE: A versatile linac-based $\mathrm{THz}$ source generating ultra-short pulses, in Proceedings of the 4th International Particle Accelerator Conference, IPAC2013, Shanghai, China, 2013 (Ref. [8]), p. 2147.

[10] M. Schuh et al., RF bunch compression studies for FLUTE, in Proceedings of the 4th International Particle 
Accelerator Conference, IPAC-2013, Shanghai, China, 2013 (Ref. [8]), p. 2144.

[11] M. Schuh et al., Status of FLUTE, in Proceedings of the 5th International Particle Accelerator Conference, Dresden, Germany, 2014 (JACoW, Geneva, Switzerland, 2014), p. 231.

[12] M. Weber et al., Start-to-end error studies for FLUTE, in Proceedings of the 36th International Free Electron Laser Conference, Basel, Switzerland, 2014 (JACoW, Geneva, Switzerland, 2015), p. 682.

[13] S. A. Mikhailov, Nonlinear electromagnetic response of graphene, Europhys. Lett. 79, 27002 (2007).

[14] A. Dienst et al., Optical excitation of Josephson plasma solitons in a cuprate superconductor, Nat. Mater. 12, 535 (2013).

[15] Y.-H. Shin, I. Grinberg, I.-W. Chen, and A. M. Rappe, Nucleation and growth mechanism of ferroelectric domain-wall motion, Nature (London) 449, 881 (2007).

[16] C. H. Back, R. Allenspach, W. Weber, S. S. P. Parkin, D. Weller, E. L. Garwin, and H. C. Siegmann, Minimum field strength in precessional magnetization reversal, Science 285, 864 (1999).

[17] K. Flöttmann, Astra-A space charge tracing algorithm, http://www.desy.de/ mpyflo.

[18] K. L. Brown, A first- and second-order matrix theory for the design of beam transport systems and charged particle spectrometers, SLAC 75, Revision 3, SLAC (1972); Report No. SLAC-PUB-3381, 1984.

[19] F. C. Iselin, The MAD program (Methodical Accelerator Design), version 8.13, physics methods manual, Report No. CERN/SL/92, 1994.

[20] M. Dohlus, T. Limberg, and P. Emma, Bunch compression for linac-based FELs, ICFA Beam Dynamics Newsletter,
Vol. 38, edited by W. Chou and I. S. Ko (Beam Dynamics Panel of the International Committee for Future Accelerators, Fermilab (Batavia, Illinois, USA), 2005), http:// icfa-usa.jlab.org/archive/newsletter/icfa_bd_nl_38.pdf.

[21] S. Fartoukh, Méthodes d'analyse d'une ligne de focalisation finale dans le cadre du projet du collisionnneur linéaire TESLA (unpublished).

[22] L. Deniau and M. Giovannozzi (private communication), http://svnweb.cern.ch/world/wsvn/madx/trunk/madX/src/ twiss.f90.

[23] M. Dohlus and T. Limberg, CSRtrack (2013), http://www .desy.de/xfel-beam/csrtrack.

[24] B. E. C. Koltenbah, C. G. Parazzoli, R. B. Greegor, and D. H. Dowell, PARMELA_B: A new version of PARMELA with coherent synchrotron radiation effects and a finite difference space charge routine, Nucl. Instrum. Methods Phys. Res., Sect. A 487, 249 (2002).

[25] M. Reiser, Theory and Design of Charged Particle Beams, 2nd ed. (Wiley VCH, New York, 2008).

[26] E. L. Saldin, E. A. Schneidmiller, and M. V. Yurkov, Longitudinal space charge-driven microbunching instability in the TESLA Test Facility linac, Nucl. Instrum. Methods Phys. Res., Sect. A 528, 355 (2004).

[27] D. Sagan, An efficient formalism for simulating the longitudinal kick from coherent synchrotron radiation, in Proceedings of the 10th European Particle Accelerator Conference, Edinburgh, Scotland, 2006 (EPS-AG, Edinburgh, Scotland, 2006), p. 2829.

[28] Y.S. Derbenev, J. Rossbach, E. L. Saldin, and V. D. Shiltsev, Microbunch radiative tail-head interaction, TESLA FEL-Report No. 1995-05.

[29] D. Diver, A Plasma Formulary for Physics, Technology and Astrophysics, 1st ed. (Wiley VCH, Berlin, 2001). 